\title{
Structure of Ba-Ti-Al-O glasses produced by aerodynamic levitation and laser heating
}

\author{
Pinit Kidkhunthod, ${ }^{1,2}$ Lawrie B. Skinner, ${ }^{1,3}$ Adrian C. Barnes, ${ }^{1}$ Wantana Klysubun, ${ }^{2}$ and Henry E. Fischer ${ }^{4}$ \\ ${ }^{1}$ H.H. Wills Physics Laboratory, University of Bristol, Tyndall Avenue, Bristol, BS8 1TL, United Kingdom \\ ${ }^{2}$ Synchrotron Light Research Insititute, 111 University Avenue, Muang, Nakhon Ratchasima, 30000, Thailand \\ ${ }^{3}$ Mineral Physics Institute, State University of New York, Stony Brook, New York 11794-2100, USA \\ ${ }^{4}$ Institut Laue-Langevin, 6 Rue Jules Horowitz, BP 156, F-38042, Grenoble Cedex 9, France
}

(Received 11 July 2014; revised manuscript received 4 August 2014; published 24 September 2014)

\begin{abstract}
$\mathrm{Ba}_{0.09} \mathrm{Al}_{0.18} \mathrm{Ti}_{0.12} \mathrm{O}_{0.61}$ glasses have been produced by aerodynamic levitation and laser heating. Neutron diffraction, $\mathrm{x}$-ray diffraction, $\mathrm{x}$-ray absorption spectroscopy, molecular dynamics simulation, and reverse Monte Carlo refinement methods have been used to obtain a detailed atomistic structural model of the glass. This model has been used to investigate its atomic coordination and network structure. It is found that the $\mathrm{Al}$ atoms are almost exclusively fourfold tetrahedrally coordinated to oxygen atoms. In contrast, the $\mathrm{Ti}$ atoms coordinate to oxygen atoms in approximately equal numbers of four- and fivefold coordinated sites with a small number of sixfold sites. The results show the presence of some tetrahedral $\mathrm{TiO}_{4}$ structural motifs although the dominant O-Ti-O bond angle occurs at $90^{\circ}$. It is found that $\mathrm{Al} / \mathrm{Ti}-\mathrm{O}$ network structure shows strong similarities with other oxide glass forming systems although a first sharp diffraction peak is not observed. The results are used to discuss the unusual properties of the Ba-Al-Ti-O glasses produced under different quenching conditions.
\end{abstract}

DOI: 10.1103/PhysRevB.90.094206

PACS number(s): 61.05.cp, 61.05.fg, 61.43.Bn, 61.43.Fs

\section{INTRODUCTION}

Barium aluminotitanate glasses are of interest due to physical properties that lend them to potential applications, for example, in the encapsulation of radionucleides in highly active nuclear waste [1-4], high refractive index materials for use in high-capacity optical disk storage systems [5], broadband photoluminescent materials [6], and as precursors for glass-ceramics for optical [7] and ferroelectric [8] applications. Further applications may arise from the ability to control the local nucleation and growth of crystals to form patterned glass ceramics by systematic annealing of the glass through, for example, laser treatment.

The aerodynamic and laser heating technique [9] is useful for the production of high melting point glasses. With this technique materials can be melted, held at very high temperatures (in excess of $2000 \mathrm{~K}$ ), and then cooled rapidly to room temperature. The absence of a container, the instantaneous cutoff of the laser heating, the small sample size, and the continuous flow of cool gas around the levitated liquid ensure that rapid quench rates (typically of the order of $500 \mathrm{~K} \mathrm{~s}^{-1}$ ) are readily obtained. As there is no container, inhomogeneous crystal nucleation [10] at the sample container surface is avoided, which allows novel glasses to form at moderate cooling rates. New aluminate and titanate glasses have been produced by this method where traditionally, bulk quenching methods have generally failed [11].

In recent work, we have used this technique to make and study glasses from mixtures of barium oxide $(\mathrm{BaO})$ and aluminiuim oxide $\left(\mathrm{Al}_{2} \mathrm{O}_{3}\right)$ [11]. In this paper, we consider the addition of $\mathrm{TiO}_{2}$ to these glasses, particularly,

Published by the American Physical Society under the terms of the Creative Commons Attribution 3.0 License. Further distribution of this work must maintain attribution to the author(s) and the published article's title, journal citation, and DOI. at the nominal equimolar composition $\mathrm{BaO}: \mathrm{Al}_{2} \mathrm{O}_{3}: \mathrm{TiO}_{2}$ $\left(\mathrm{Ba}_{0.09} \mathrm{Al}_{0.18} \mathrm{Ti}_{0.12} \mathrm{O}_{0.61}\right)$. At this composition, the optical properties of the glass are very sensitive to the precise rate at which the liquid is quenched [10]. In particular, when the liquid is quenched at the maximum rate $\left(500 \mathrm{~K} \mathrm{~s}^{-1}\right)$ the glass always appears black and opaque. In contrast, when the quench rate is more moderate and carefully controlled it is possible to make, from the same starting liquid, a clear and optically transparent glass. At slightly slower quench rates, a turbid, but apparently still noncrystalline glass, is produced. The origin of this turbidity is unclear but is thought to arise either from strong local density fluctuations in the glass or due to the formation of nanocrystalline inclusions.

The reasons for the black opaque appearance of the glasses produced by rapid quenching is not clear; the effect is not seen in pure barium aluminate glass. A similar coloration has been reported in glasses containing barium titanate [12] and is attributed to the partial reduction of some of the titanium centers at four- and sixfold coordinated sites. We are particularly interested to determine the $\mathrm{Ti}$ coordination and any evidence for a change of oxidation state of $\mathrm{Ti}$ in our glasses as we believe this will provide further insight into this behavior.

The formation of turbid glasses on cooling under similar conditions has also been observed in glassy yttria aluminate and its related rare-earth (e.g., erbium, holmium) analogues [13]. Detailed studies of these materials over wide composition ranges attribute this turbidity to polyamorphism (the microscopic separation of the liquid into phases of high- and lowdensity glass with identical chemical composition) [14] or the formation of nanocrystalline glass ceramics [10]. The establishment of true polyamorphism in these glasses remains controversial and is intimately linked with the proposal that it occurs due to a liquid-liquid transition in the liquid before vitrification takes place $[15,16]$. These properties are closely related to the medium range order (MRO) in the glass. Hence we are interested to understand why, in $\mathrm{Ba}_{0.09} \mathrm{Al}_{0.18} \mathrm{Ti}_{0.12} \mathrm{O}_{0.61}$ glass, the ( $\mathrm{Al} / \mathrm{Ti})-\mathrm{O}$ network structure gives rise to similar behavior. 
Finally, the use of this or other glasses for encapsulating radio nucleides is closely linked to how the $\mathrm{Ba}$ atoms enter the glass structure. Hence, we wish to determine how the $\mathrm{Ba}$ atoms are coordinated and incorporated into the (Al/Ti)-O glass network especially when compared to pure Ba-Al-O glasses [11].

In this paper, we present neutron and high energy x-ray diffraction data of the opaque and clear glasses. We present an analysis of the total radial distribution functions and detailed atomistic models of the glasses obtained by molecular dynamics (MD) simulation and by reverse Monte Carlo (RMC) refinement of the $\mathrm{MD}$ atomic configurations to the diffraction data. We have particularly compared the virtues of the Buckingham type potentials used in the previous study of barium aluminate glass [11,17] with the more recent Morse type potentials of Pedone et al. [18]. In addition, we present $\mathrm{x}$-ray absorption spectra (XANES and EXAFS) obtained from the glasses and compare them to the spectra predicted from our atomistic models in order to interpret more precisely the nature of the Ti coordination and oxidation state in the glasses.

\section{THEORY}

\section{A. Diffraction}

The $\mathrm{x}$-ray or neutron diffraction pattern for a disordered material may be expressed,after experimental corrections, as the total structure factor,

$$
\begin{aligned}
S(Q)-1= & \frac{1}{|\langle w(Q)\rangle|^{2}} \sum_{\alpha} \sum_{\beta} c_{\alpha} c_{\beta} w_{\alpha}^{*}(Q) w_{\beta}(Q) \\
& \times\left[S_{\alpha \beta}(Q)-1\right],
\end{aligned}
$$

where $c_{\alpha}, c_{\beta}$ are the atomic concentrations of species $\alpha$ and $\beta, w_{\alpha}(Q)$ and $w_{\beta}(Q)$ are their (complex) scattering factors, $Q$ is the magnitude of the scattering vector and $S_{\alpha \beta}(Q)$ is the Faber-Ziman partial structure factor for correlations between $\alpha$ and $\beta$ [19].

For neutrons, $w_{\alpha}(Q)$ is the $Q$ independent neutron coherent scattering length $b_{\alpha}$, which is a real number for $\mathrm{Ba}, \mathrm{Ti}, \mathrm{Al}$, and $\mathrm{O}$ at thermal neutron energies. The values of $b_{\alpha}$ used in this work were taken from the Neutron data booklet [20] and are listed in Table I and we denote the neutron total structure factor as $S_{N}(Q)$.

For $\mathrm{x}$ rays, $w_{\alpha}(Q)$ is usually expressed as $w_{\alpha}(Q)=$ $f_{\alpha}(Q)+f_{\alpha}^{\prime}\left(E_{0}\right)+f_{\alpha}^{\prime \prime}\left(E_{0}\right)$, where $f_{\alpha}(Q)$ is the form factor for species $\alpha$ and is related to the spherically averaged electron density for the species. $f_{\alpha}^{\prime}\left(E_{0}\right)$ and $f_{\alpha}^{\prime \prime}\left(E_{0}\right)$ are dispersion terms (commonly referred to as the anomalous scattering terms) that

TABLE I. The neutron scattering parameters used for correction and analysis of the neutron diffraction results [20].

\begin{tabular}{lccc}
\hline \hline Atom & $\begin{array}{c}\text { Coherent scattering } \\
\text { length (fm) }\end{array}$ & $\begin{array}{c}\text { Total Scattering } \\
\text { cross-section (barn) }\end{array}$ & $\begin{array}{c}\text { Absorption } \\
\text { cross-section (barn) }\end{array}$ \\
\hline $\mathrm{Ba}$ & $5.07(3)$ & $3.38(10)$ & $1.1(1)$ \\
$\mathrm{Ti}$ & $-3.370(13)$ & $4.35(3)$ & $6.09(13)$ \\
$\mathrm{Al}$ & $3.449(5)$ & $1.503(4)$ & $0.231(3)$ \\
$\mathrm{O}$ & $5.805(4)$ & $4.232(6)$ & $0.00019(2)$ \\
\hline \hline
\end{tabular}

are a result of changes in scattering due to $\mathrm{x}$-ray resonance near an atomic absorption edge. In this work, neutral atom form factors [21] were used in all calculations and analysis and we denote the x-ray total structure factor as $S_{X}(Q)$. At $Q=0$, these have a value identical to the atomic number of the species in question. The dispersion terms were calculated at the incident $\mathrm{x}$-ray energy using the FPRIME programme [22].

The partial structure factors $S_{\alpha \beta}(Q)$ are directly related to the real-space partial radial distribution functions $g_{\alpha \beta}(r)$ by the sine Fourier transform relation

$$
g_{\alpha \beta}(r)-1=\frac{1}{2 \pi^{2} \rho r} \int_{0}^{\infty} Q\left[S_{\alpha \beta}(Q)-1\right] \sin (Q r) d Q .
$$

The real-space total pair distribution function $G(r)$ is obtained from $S(Q)$ by the sine Fourier transform relation,

$$
G_{(N / X)}(r)-1=\frac{1}{2 \pi^{2} \rho r} \int_{0}^{\infty} Q\left[S_{(N / X)}(Q)-1\right] \sin (Q r) d Q,
$$

where $\rho$ is the atomic number density of the material. For neutron scattering the weighting of the partial structure factors are independent of $Q$ so that $G_{N}(r)$ may be expressed directly by a weighted sum of the partial radial distribution functions as

$$
G_{N}(r)-1=\sum_{\alpha} \sum_{\beta} c_{\alpha} c_{\beta} b_{\alpha} b_{\beta}\left[g_{\alpha \beta}(r)-1\right] .
$$

For the case of $G_{X}(r)$, this separation into separate terms is not possible as each $g_{\alpha \beta}$ has a different $Q$ dependence. For the case of an isolated peak in $G_{X}(r)$, it is possible to construct a modified $S_{X}(Q)$ in which the relevant partial partial structure factor is given a $Q$ independent weight so that it Fourier transforms identically to that for the case of neutron scattering. In the case of Ba-Al-O glass [11], this was used to compare directly the neutron and x-ray data for the first Al-O peak in $\left.G_{(} r\right)$. A similar separation is not possible in this study due to the close Al-O (1.8 $⿱$ ) and Ti-O (2.0 $)$ bond distances. The situation is further complicated as the coherent neutron scattering length of $\mathrm{Ti}$ is negative such that the Ti-O peak in $G_{N}(r)$ has a negative weight leading to a degree of cancellation with the Al-O peak at shorter distance.

As a consequence, the amount of information that can be obtained directly from $G_{N}(r)$ and $G_{X}(r)$ is limited and restricted to peak fitting procedures. In this study, we have therefore concentrated on comparing and fitting the experimental diffraction data to that generated from structural models derived from MD modeling techniques.

\section{B. Structure modelling}

An alternative approach to the direct real-space analysis of diffraction data by Fourier transform is to compare the experimental $S_{N / X}(Q)$ and $G_{N / X}(r)$ with a calculation of the corresponding functions obtained from atomistic models of the material. In this case, the $Q$ dependent weighting factors that occur in the $\mathrm{x}$-ray data may be directly included in the model calculation without needing to deconvolve them in a reciprocal to real-space transformation of the experimental data. 
There are a variety of ways in which theoretical atomistic models may be obtained including, ab initio MD [23,24], MD, and Monte Carlo [25] methods. All of these methods produce, after suitable equilibration time, a series of atom configurations from which $g_{\alpha \beta}(r)$ may be directly calculated and averaged. These may then be weighted and combined to make a direct comparison with the experimental $G_{N / X}(r)$ or used to calculate the $S_{\alpha \beta}(Q)$ according to Eq. (1) or (3) and hence to make a direct comparison with the data.

While ab initio methods deal well with short-range chemical bonding they are generally restricted to a few hundred atoms making it difficult to simulate glasses that often show strong medium range order. In contrast, while MD and Monte Carlo methods based on pair potentials can include tens of thousands of atoms they often fail to simulate well the effects of the strong three-body forces characteristic of network glasses. In addition, none of these methods are able to quench the simulation of the liquid to a glass on experimental timescales, so that simulations have high (compared to the real glass) fictive temperatures, a smeared out glass transition and even the ability to nucleate crystals below the glass transition temperature [26,27].

However, in our previous work on Ba-Al-O glasses [11] we found that MD calculations using simple modified Coulomb pair potentials gave results that are in reasonable agreement with the experimental data. These provided an excellent starting point for the refinement of the structure allowing us to eliminate some of the shortcomings noted above. In this study we have carried out MD calculations using two sets of potentials (Buckingham [17,28] and Morse [18,29]) in order to establish which produce the best agreement with experiment and hence are most suitable for further structural refinement.

\section{Structural model refinement}

A good agreement of the diffraction data with the molecular dynamics simulations indicates that the simulation configuration is a good representation of the structure of the material under study. However, perfect agreement between simulation and data is rare and dependent on the simulation method used. Simple MD simulations using pair potentials are fast but, as noted above and especially for the case of oxide glasses, often fail to produce structural features characteristic of three-body interactions such as strong local tetrahedral motifs. However, they have proved to be a good starting point to refine the atomistic structure to the experimental data $[11,30]$.

In this study, we use the reverse Monte Carlo (RMC) technique [31,32] to refine our structural models obtained from MD simulations. In this method, the structure calculated from the starting atomistic configuration is compared with the experimental data. Subsequently, atoms in the configuration are randomly moved short distances and the configuration compared with the experimental data again. Moves that improve the fit to the data (measured as $\chi^{2}$ ) are accepted. Moves that worsen the fit are accepted with a probability proportional to the change in $\exp \left(-\Delta \chi^{2}\right)$ (the equivalent to the Boltzmann factor in normal Monte Carlo simulations).

In this work we specifically use the term "RMC refinement" rather than "RMC modeling" to emphasise that we are assuming the MD simulation has captured the broad structure of the material based on realistic interatomic potentials. We justify that it is a refinement by making small displacements in the RMC step, observing fast convergence and verifying that the typical displacements of the atoms from the initial position after refinement are much less than the typical interatomic separation (i.e., $\ll \sim 0.5 \AA$ ). Nevertheless, note that the RMC refinement is not guided by and does not take into account the original interatomic potentials.

\section{X-ray absorption spectroscopy (XAS)}

The $\mathrm{x}$-ray absorption edge of an atom in a condensed matter material is modified from the isolated atom absorption edge due to the back scattered interference of the ejected photo electron with the atoms surrounding the target atom. This interference leads to oscillations in both $f^{\prime}(E)$ and $f^{\prime \prime}(E)$ around the absorption edge of the element. For the case of $f^{\prime \prime}(E)$ this is reflected directly in the X-ray absorption spectrum $[\mu(E)]$ of the material in this region [33].

The absorption is generally separated into different regions. The EXAFS region extends from energies of a few $\mathrm{eV}$ from the edge up to $\sim 500-1000 \mathrm{eV}$ from the edge. In the first order theory, the oscillations observed in $\mu(E)$ are directly related to the distances of the neighboring atoms to the target atom by Fourier transform after correction for phase shifts in the photoelectron scattering. In a more rigorous analysis, the photoelectron scattering is calculated for model local configurations around the target atom and all the single and multiple scattering paths are calculated and refined to fit the measured spectra [34,35]. For crystalline materials, the multiple scattering terms may be significant. In contrast, for disordered materials, the issues are more concerned with how to properly average all the local configurations in order to compare to the experimental data. These issues are thoroughly discussed by Filipponi [36]. An alternative strategy, which we employ in this work, is to calculate the average of the EXAFS signal from all the target atoms in our MD and RMC configurations and compare this directly to the measured spectra.

The region close to the edge is characterised by interference of the back scattered photo-electron on length scales greater than the typical interatomic distance (XANES). The calculations in this region are difficult and dominated by multiple scattering [34]. However, in the particular case of Ti very strong features in the pre-edge region for the $\mathrm{Ti} K$ edge are characteristic of both the oxidation state and local configuration of the $\mathrm{Ti}$ atoms in the structure. The origin of these features can be directly calculated or verified by comparison to the features in the local Ti coordination known from crystal XANES measurements [37].

\section{EXPERIMENTAL METHOD}

\section{A. Sample preparation}

Finely powdered $\mathrm{BaTiO}_{3}(99.995 \%)$ and $\mathrm{Al}_{2} \mathrm{O}_{3}(99.99 \%)$ were dried in air in a furnace at $1273 \mathrm{~K}$ for 24 hours. Equimolar quantities were carefully mixed, placed on a copper hearth and rapidly melted using a $\mathrm{CO}_{2}$ laser to form homogeneous small polycrystalline beads with a diameter of $\sim 2 \mathrm{~mm}$ and a mass of $\sim 20 \mu \mathrm{g}$. The samples were levitated on an ultra 
TABLE II. The atomic composition of the as prepared glasses as determined by wavelength dispersive EPMA analysis. The relative error on the metal concentrations is better than $1 \%$. The oxygen concentration was determined assuming $2+, 3+$, and $4+$ oxidation states for the $\mathrm{Ba}, \mathrm{Al}$, and $\mathrm{Ti}$ atoms respectively. No difference in composition between the clear and opaque black glasses, within error, was identified by EPMA analysis.

\begin{tabular}{lcc}
\hline \hline Atom & Nominal concentration & Concentration \\
\hline $\mathrm{Ba}$ & 0.1 & 0.09 \\
$\mathrm{Ti}$ & 0.1 & 0.12 \\
$\mathrm{Al}$ & 0.2 & 0.18 \\
$\mathrm{O}$ & 0.6 & 0.61 \\
\hline
\end{tabular}

pure (99.9999\%) Argon gas stream (BOC) in the aerodynamic levitator and melted with a $\mathrm{CO}_{2}$ laser (wavelength $10.6 \mu \mathrm{m}$ ) to reach a final pyrometric (at a wavelength of $0.9 \mu \mathrm{m}$ ) temperature of $2500 \mathrm{~K}$ (uncorrected for emissivity). Assuming an emissivity of $e=0.9$ this corresponds to an actual temperature of $\sim 2550 \mathrm{~K}$ [38]. The samples were allowed to mix and equilibrate for 2 minutes and then quenched in the argon gas flow by either switching the laser power off instantaneously or by controlled decrease of the laser power under computer control. For a typical sample of 2-mm diameter the maximum quench rate achieved was $\sim 500 \mathrm{~K} \mathrm{~s}^{-1}$ at $2500 \mathrm{~K}$. At this quench rate, opaque black glass beads were made. Clear and transparent glass beads were obtained when the quench rate was reduced to $\sim 50 \mathrm{~K} \mathrm{~s}^{-1}$ at $2500 \mathrm{~K}$. The density of both the clear and opaque glass was $3.9 \pm 0.1 \mathrm{~g} \mathrm{~cm}^{-1}$ as measured by Archimede's displacement method.

Some mass loss in the samples was observed after they had been prepared. Similar effects have been observed previously in samples of pure barium aluminate glass [11] leading to a change from the target composition. We therefore carried out a wavelength dispersive microprobe analysis (EMPA) of sample beads in order to obtain the composition of the beads. The results are shown in Table II. In this paper, for brevity, we shall henceforth refer to this glass composition as BATO glass.

\section{B. Neutron diffraction}

Neutron diffraction experiments were carried out using the D4C diffractometer at the Institut Laue-Langevin, Grenoble, France [39]. An incident wavelength of 0.4965 (1) A, giving an experimental $Q$ range of $\sim 0.3<Q<23.0 \AA^{-1}$, was used for all measurements. Approximately 50 glass beads were packed into a thin walled vanadium can of $4.8 \mathrm{~mm}$ internal diameter and wall thickness of $0.1 \mathrm{~mm}$. Measurements were made for both the rapidly quenched, black glasses, and the slowly quenched transparent glasses. The total amount of the sample in the neutron beam was estimated from the density of the glass and the packing density of the spheres in the container (as measured by the total height of the spheres). The experimental data were corrected for self-absorption, multiple scattering and container scattering [40]. The sample scattering was put on an absolute scale by reference to a standard vanadium bar of $6.072(6) \mathrm{mm}$ placed at the sample position.

The instrument stability was continually monitored by carrying out repeated scans of 2 hours over the whole angular range. No variation in intensity was observed in these scans, except that expected from the counting statistics. The data collection time for each sample was approximately 12 hours in total. Due to the uncertainties in the packing fraction a final small empirical scaling correction $(<2 \%)$ was made to the measured scattering to ensure that the high $Q$ scattering limit was consistent with the known scattering cross-sections of the sample elements. The neutron scattering scattering parameters used for the data corrections and analysis are show in Table I.

\section{X-ray diffraction}

High energy diffraction on the black and transparent glasses was carried out at Beamline 11-1DC at the Advanced Photon Source (APS), Argonne National Laboratory, USA. The incident wavelength was 0.10803(1) $\AA$ corresponding to an incident energy of 114.78(1) keV. The cross-sectional area of the beam was $0.5 \times 0.5 \mathrm{~mm}^{2}$. Samples were held in the incident $\mathrm{x}$-ray beam using kapton tape attached to the top and bottom of the sample spheres such that the incident and diffracted beams passed only through the sample. At this energy the absorption length in the sample is $37 \mathrm{~mm}$ and corresponds to $\sim e q 94 \%$ transmission through the thickest part of the sample. A Perkin Elmer XRD1621 area detector, with a 1-mm brass sheet covering the active area to reduce background scattering due to $\mathrm{Ba}$ fluorescence, was placed at $268 \mathrm{~mm}$ from the sample. The 2D diffraction patterns were centered, corrected for polarization and converted into 1D patterns using the FIT2D program [41]. The wavelength calibration was made with respect to a standard $\mathrm{CeO}_{2}$ sample placed at the sample position. The resulting patterns were corrected for multiple scattering, absorption and Compton scattering and normalised by fitting to the atomic form factors at high $Q$ values using standard procedures $[19,42]$. The corrected $S_{X}(Q)$ covers the range $0.5<Q<\sim 20 \AA^{-1}$.

\section{XAS measurements}

$\mathrm{X}$-ray absorption spectroscopy (XAS) measurements at the Ti $K$ edge were carried out at in transmission mode using the BL8 beamline at the Siam photon source at the Synchrotron Light Research Institute, (SLRI), Thailand [43]. An incident beam size of $13 \mathrm{~mm}$ width by $1 \mathrm{~mm}$ height, energy $4900 \mathrm{eV}<E<5300 \mathrm{eV}$ was obtained by Rocking a Ge(220) double crystal monochromator giving and energy resolution of $\Delta E / E=3 \times 10^{-4}$. The photon energy calibration was carried out with a Ti foil with a reference $K$-edge energy of 4,996.0(2) eV [44].

The sample was prepared by grinding the glass beads into very fine powders of particle size $\leqslant 1 \mu \mathrm{m}$ using an agate pestle and mortar. The fine powder was compacted between two layers of kapton tape separated by a polyimide spacer to form a uniform sample of $35 \mu \mathrm{m}$ thickness. This thickness is the optimum path length for the transmission EXAFS measurements. The incident x-ray intensity was measured using a 10-cm-long $\mathrm{Ar} / \mathrm{He}$ ionization chamber with argon and helium pressures of 15 and 998 mbar respectively. The transmitted intensity was measured using a 40-cm-long $\mathrm{Ar} / \mathrm{He}$ ionization chamber with argon and helium pressures of 80 and 933 mbar respectively. Two EXAFS scans of 15 minutes duration were made for each sample to verify the detector 
stabilities. No deviations apart from the expected statistical variations due to the counting statistics were observed.

The XAS spectra (XANES and EXAFS) were normalized and corrected to give $\chi(k)$ using the ATHENA program [45] with an automatic background correction. The spectra were analyzed in detail using IFEFFIT [45] in combination with FEFF8 [34].

\section{MODELING METHODS}

\section{A. MD simulation}

MD simulations were carried out for BATO glass with the DL_POLY (version 2.17) MD program [46]. The simulation box contained $453 \mathrm{Ba}, 906 \mathrm{Al}, 604 \mathrm{Ti}$, and 3,020 $\mathrm{O}$ atoms to give the experimentally determined atomic fractions (Table II). Two sets of potentials were used: Buckingham potentials of the form

$$
\phi_{i j}\left(r_{i j}\right)=A_{i j} \exp \left(-\frac{r_{i j}}{\rho_{i j}}\right)-\frac{C_{i j}}{r_{i j}^{6}}+\frac{q_{i} q_{j}}{4 \pi \epsilon_{0} r},
$$

with the potential parameters taken from Bush et al. [17] as used in the analysis of Ba-Al-O glass [11], and Morse potentials of the form

$$
\phi_{i j}\left(r_{i j}\right)=D_{i j}\left(\left\{1-\exp \left[-\left(\frac{a_{i j}}{r_{i j}-r_{0}}\right)\right]\right\}^{2}-1\right)+\frac{C_{i j}}{r_{i j}^{12}},
$$

with the potential parameters taken from Pedone et al. [18]. The parameters for the potentials are show in Table III . Apart from O-O, all the like-like interactions were assumed to be purely Coulombic in nature.

The MD simulations runs were started from a random atomic configuration in which the interatomic distances were set according to minimum distance chemical constraints determined from bond distances observed in crystalline materials. The simulation box was a cube of $41.44 \AA$, corresponding to an atomic number density of $0.07 \AA^{-3}$ obtained from the measured sample density of $3.9 \mathrm{~g} \mathrm{~cm}^{-3}$. The simulation was

\begin{tabular}{|c|c|c|c|c|}
\hline \multicolumn{5}{|c|}{ Morse potentials according to Pedone et al. [18] } \\
\hline Pair & $\begin{array}{c}D_{i j} \\
(\mathrm{eV})\end{array}$ & $\begin{array}{c}a_{i j} \\
\left(\AA^{-2}\right)\end{array}$ & $\begin{array}{c}r_{o} \\
(\AA)\end{array}$ & $\begin{array}{c}C_{i j} \\
\left(\mathrm{eV} \AA^{12}\right)\end{array}$ \\
\hline $\mathrm{Ba}^{1.2}-\mathrm{O}^{-1.2}$ & 0.065011 & 1.547596 & 3.393410 & 5.0 \\
\hline $\mathrm{Ti}^{2.4}-\mathrm{O}^{-1.2}$ & 0.024235 & 2.254703 & 2.708943 & 1.0 \\
\hline $\mathrm{Al}^{1.8}-\mathrm{O}^{-1.2}$ & 0.361581 & 1.900442 & 2.164818 & 0.9 \\
\hline $\mathrm{O}^{-1.2}-\mathrm{O}^{-1.2}$ & 0.042395 & 1.379316 & 3.618701 & 22.0 \\
\hline \multicolumn{5}{|c|}{ Buckingham potentials according to Bush et al. [17] } \\
\hline Pair & $\begin{array}{c}A_{i j} \\
(\mathrm{eV})\end{array}$ & & $\begin{array}{l}\rho_{i j} \\
(\AA)\end{array}$ & $\begin{array}{c}C_{i j} \\
\left(\mathrm{eV} \AA^{6}\right)\end{array}$ \\
\hline $\mathrm{Ba}^{2}-\mathrm{O}^{-2}$ & 4818.416 & & 0.3067 & 0.00 \\
\hline $\mathrm{Ti}^{4}-\mathrm{O}^{-2}$ & 2088.107 & & 0.2888 & 0.00 \\
\hline $\mathrm{Al}^{3}-\mathrm{O}^{-2}$ & 2409.505 & & 0.2649 & 0.00 \\
\hline $\mathrm{O}^{-2}-\mathrm{O}^{-2}$ & 25.410 & & 0.6937 & 32.32 \\
\hline
\end{tabular}

TABLE III. The potential parameters used for the MD simulations. carried out using an NVT Berendsen thermostat [25]. The simulation run was started at $2500 \mathrm{~K}$ and ran with a time step of $0.001 \mathrm{ps}$. The system was equilibrated for 500 steps and then run for an additional 50000 steps. After this, the simulation temperature was set to $300 \mathrm{~K}$ and the process repeated. The final configuration was saved as the starting configuration for the structural refinement of the glass.

In addition, further MD runs around this composition were made using the Pedone et al. potentials to check for the effect of uncertainties in our measured composition (see Table II). It was found that the results did not alter our conclusions and hence our detailed analysis was confined to the measured composition.

\section{B. Reverse Monte Carlo refinement}

The final MD atomic configurations were refined using the RMCPRFILE [32] (Version 6) program to obtain atomistic models that were consistent with the diffraction data. Initially, $S_{X}(Q)$ and $S_{N}(Q)$ were used as the input data for the refinement. Nearest-neighbor cutoff distances determined from the shortest distance of approach in the MD simulations were imposed for each $g_{\alpha \beta}(r)$. However, with these experimental constraints alone it was found that an unphysical "pile up" of some bonds at these cutoff distances, primarily in $g_{\mathrm{BaO}}(r)$, arose during the refinement procedure. We believe this behavior is associated with the observation that a small movement in $r$ of an atom in the RMC refinement is associated with a very small change over the entire $Q$ range in $S_{N / X}(Q)$. As a consequence, physically unfavorable moves still have a high probability of being accepted, especially in the case of many-atom systems and a limited number of data sets. In contrast, comparison to the data in real space is more sensitive to the direct changes caused by the real-space translation of the atom in the simulation box. For these reasons, we also included $G_{N}(r)$ data as a further constraint in our refinement even though it does not contain any more information than $S_{N}(Q)$. With this procedure unphysical peaks in $g_{\alpha \beta}(r)$ were eliminated without the need for any further constraints.

The RMC refinement was run with a $0.01-\AA$ maximum step until the overall $\chi^{2}$ fit reached a constant value. In practice, this was found to occur after about 48 hours running on a PC (Intel i3/2.8GHz). At this point, the $\chi^{2}$ had become almost constant. At the end of the refinement, the mean square displacement of each atom from its starting position was calculated to verify that the atoms had moved much less than an atomic diameter during the refinement cycle. To improve the configuration statistics, the refinement procedure was repeated several times using a different random number seed. The analysis of the atomic configurations was carried out by averaging the results from the configurations obtained.

\section{RESULTS}

\section{A. Diffraction results}

The measured neutron and $\mathrm{x}$-ray diffraction patterns are shown in Fig. 1. No difference within statistical errors could be observed in the diffraction patterns from the black and the transparent glasses. 

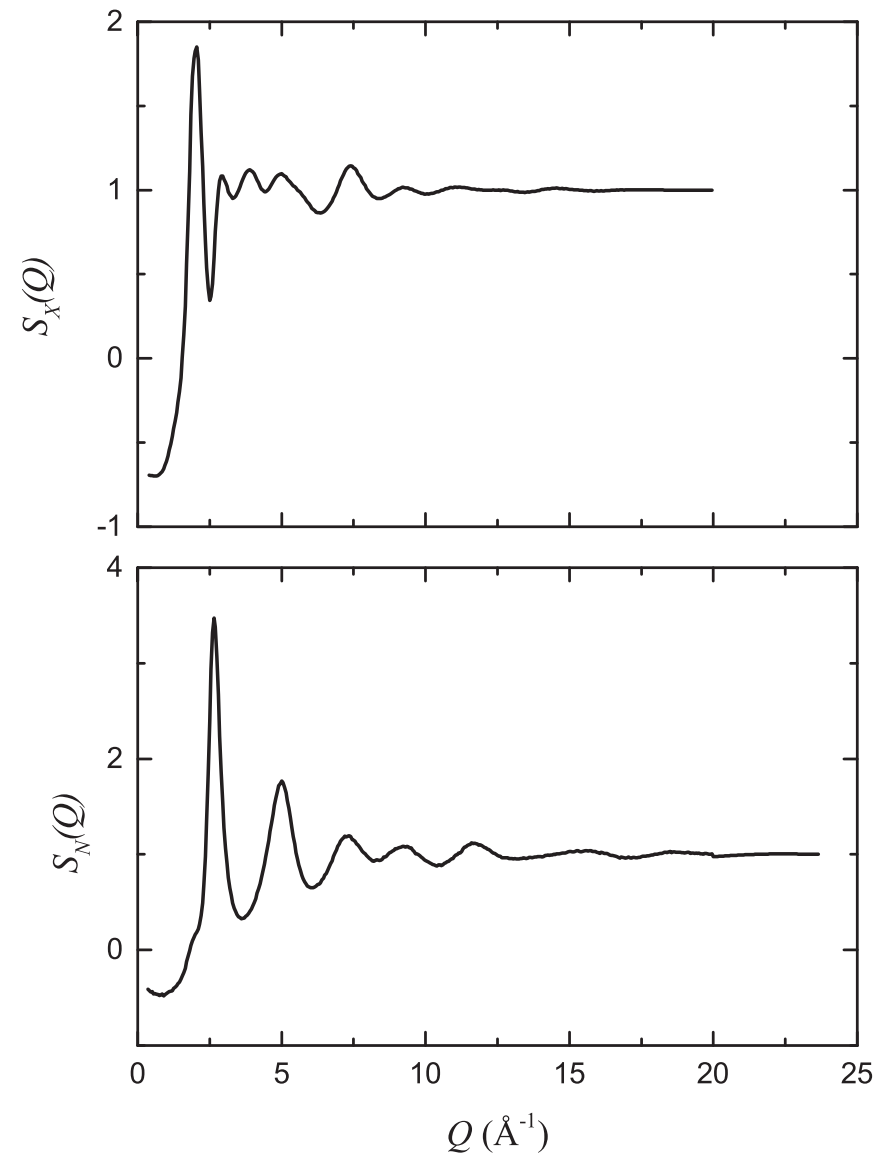

FIG. 1. The experimentally determined structure factors, $S_{N}(Q)$ and $S_{X}(Q)$. The maximum value $Q_{\max }$ is $23.5 \AA^{-1}$ for $S_{N}(Q)$ and $20 \AA^{-1}$ for $S_{X}(Q)$. The statistical difference between the diffraction patterns for the black and transparent glasses is not observable at the resolution of these figures.

$S_{N}(Q)$ shows a first peak at 2.65(5) $\AA^{-1}$ with evidence of a small prepeak at $\sim 2.0 \AA^{-1}$. There is also a small rise in the scattering, a low $Q$ below $\sim 1 \AA^{-1}$. $S_{X}(Q)$ shows a first peak at $\sim 2.0 \AA^{-1}$ that on closer inspection appears to be composed of two closely overlapping peaks at $\sim 1.95$ and $\sim 2.05 \AA^{-1}$, respectively. There is also a very weak indication of a small rise at low $Q$ in a similar region to that observed in $S_{N}(Q)$.

The total pair distribution functions, $G_{N}(r)$ and $G_{X}(r)$ are plotted in Fig. 2. $G_{X}(r)$ shows a single peak at 1.80(5) $\AA$ that is associated with the overlapping and unresolved nearestneighbor $\mathrm{Al}-\mathrm{O}$ and $\mathrm{Ti}-\mathrm{O}$ distances expected at 1.7-1.8 and 1.8-2.0 А, respectively.

In contrast, $G_{N}(r)$ shows a nearest-neighbor peak at $1.75(2) \AA$ followed by a negative peak at 1.97(2) $\AA$. The negative peak in $G_{N}(r)$ is associated with the nearest-neighbor Ti-O correlations and arises due to the negative scattering length of $\bar{b}_{\mathrm{Ti}}=-3.370 \mathrm{fm}$ (see Table I). However, the overlap of the Al-O and Ti-O peaks in both $G_{X}(r)$ and $G_{N}(r)$ (where the negative Ti-O peak partially cancels the intensity of the shorter Al-O peak) means that it is not possible to obtain the oxygen coordination around $\mathrm{Al}$ and $\mathrm{Ti}$ by direct integration.

Both $G_{X}(r)$ and $G_{N}(r)$ show a strong second peak at $\sim 2.8 \AA$ that may be associated most strongly with the O-O
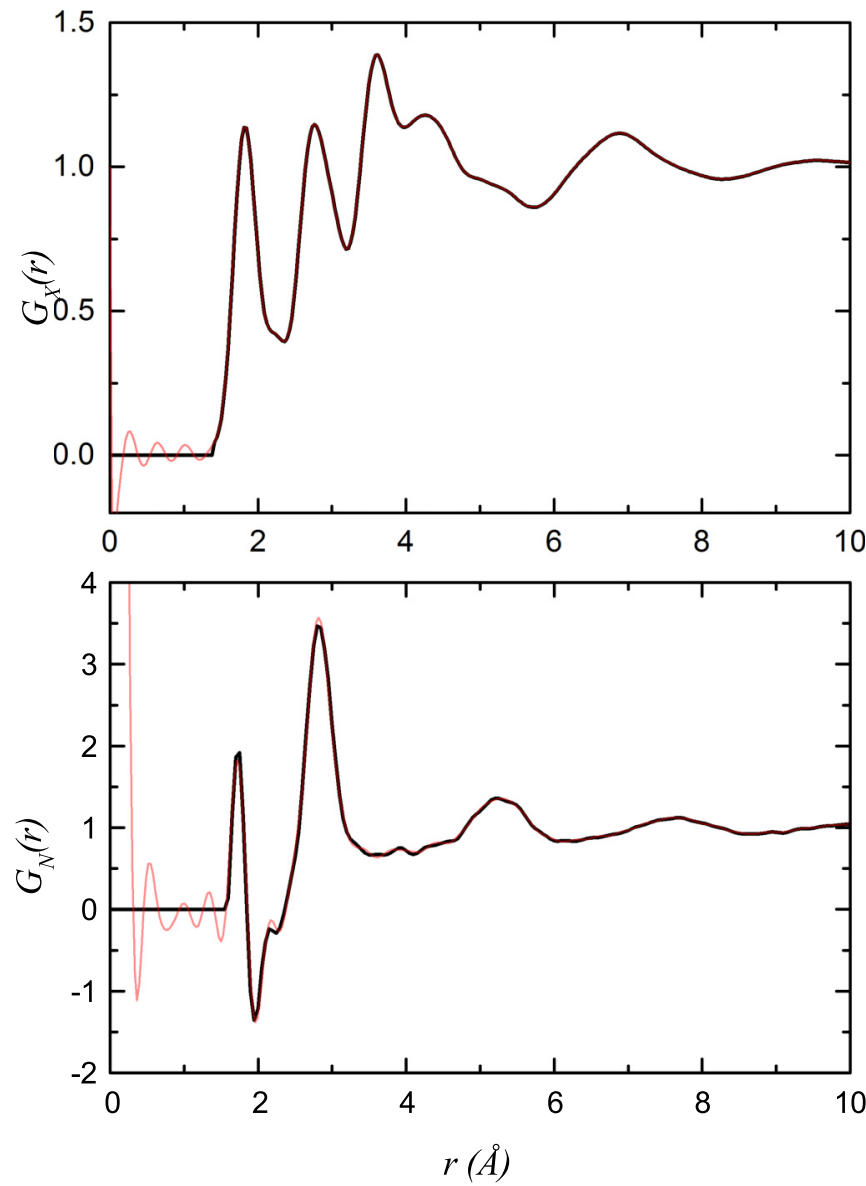

FIG. 2. (Color online) The Fourier transforms, $G_{N}(r)$ and $G_{X}(r)$ of the total structure factors shown in 1 . The solid black line shows physically real peaks and the cutoff set at low $r$. These data were back transformed to obtain $S_{N}(Q)$ and $G_{X}(r)$ used in the further analysis. The light red line shows the termination errors in the direct transform to $G_{N / X}(r)$ due to the finite $Q_{\max }$ and other systematic errors (e.g., small slopes) in the measured $S_{N / X}(Q)$.

correlations in the glass network and the nearest-neighbor Ba-O correlation as observed in other systems [11]. $G_{X}(r)$ shows strong features in the region 3.5-5.0 and $\sim 7 \AA$ that are absent in $G_{N}(r)$. Inspection of the weights in Fig. 3 shows that the $M-M(M=\mathrm{Ba}, \mathrm{Ti}, \mathrm{Al})$ and $\mathrm{Ba}-\mathrm{O}$ correlations have a strong weight in $G_{X}(r)$ while being almost absent in $G_{N}(r)$. This suggests that these peaks in $G_{X}(r)$ arise from strong nearest-neighbor $M-M$ and $\mathrm{Ba}-\mathrm{O}$ correlations. Due to the large number of overlapping peaks, we did not attempt to ascertain any coordination numbers by direct integration of peaks in either $G_{N}(r)$ or $G_{X}(r)$. The clear peaks in $G_{N}(r)$ at $\sim 5$ and $\sim 7.5 \AA$ are most likely due to $g_{\mathrm{OO}}(r)$ that contributes almost $50 \%$ of the total.

\section{B. Molecular dynamics}

Figure 4 shows the measured $Q\left(S_{N}(Q)-1\right)$ and $Q\left(S_{X}(Q)-1\right)$ compared to MD simulations based on the potentials of Bush et al. [17] and Pedone et al. [18], respectively. The data are plotted as $Q(S(Q)-1)$ to emphasise the structure at high $Q$. 


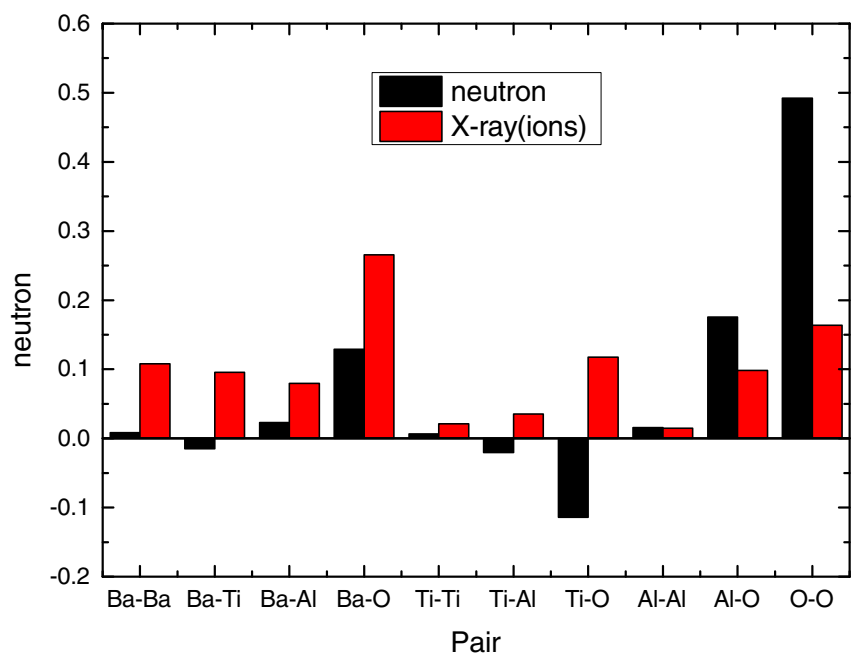

FIG. 3. (Color online) The weighting of the partial structure factors in $S_{N}(Q)$ and $S_{X}(Q)$. The x-ray weights are calculated at $Q=0$ [21]. The negative values for the neutron weights are due to the negative neutron scattering length of Ti.
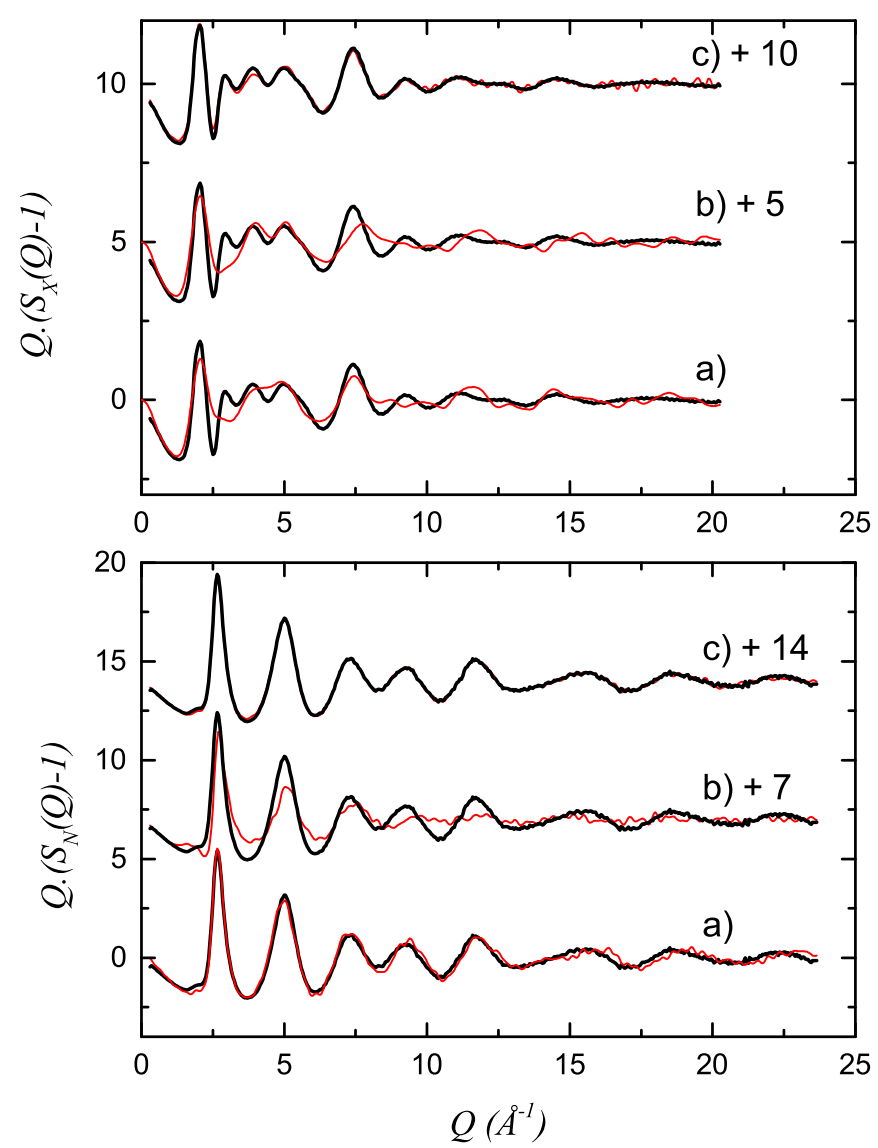

FIG. 4. (Color online) Comparison of the MD and MD-RMC results with the x-ray (upper box) and neutron (lower box) diffraction data (black lines). The data are plotted as $Q(S(Q)-1)$ in order to emphasize the fit to the data at high $Q$. (a) The MD data (red line) generated with Morse potentials [18] compared to the data. (b) The MD data (red line) generated with Buckingham potentials [17] compared to the data. (c) The MD-RMC fit (red line) to the data. Both (a) and (b) converged to the same MD-RMC result (c) within the resolution of this figure.
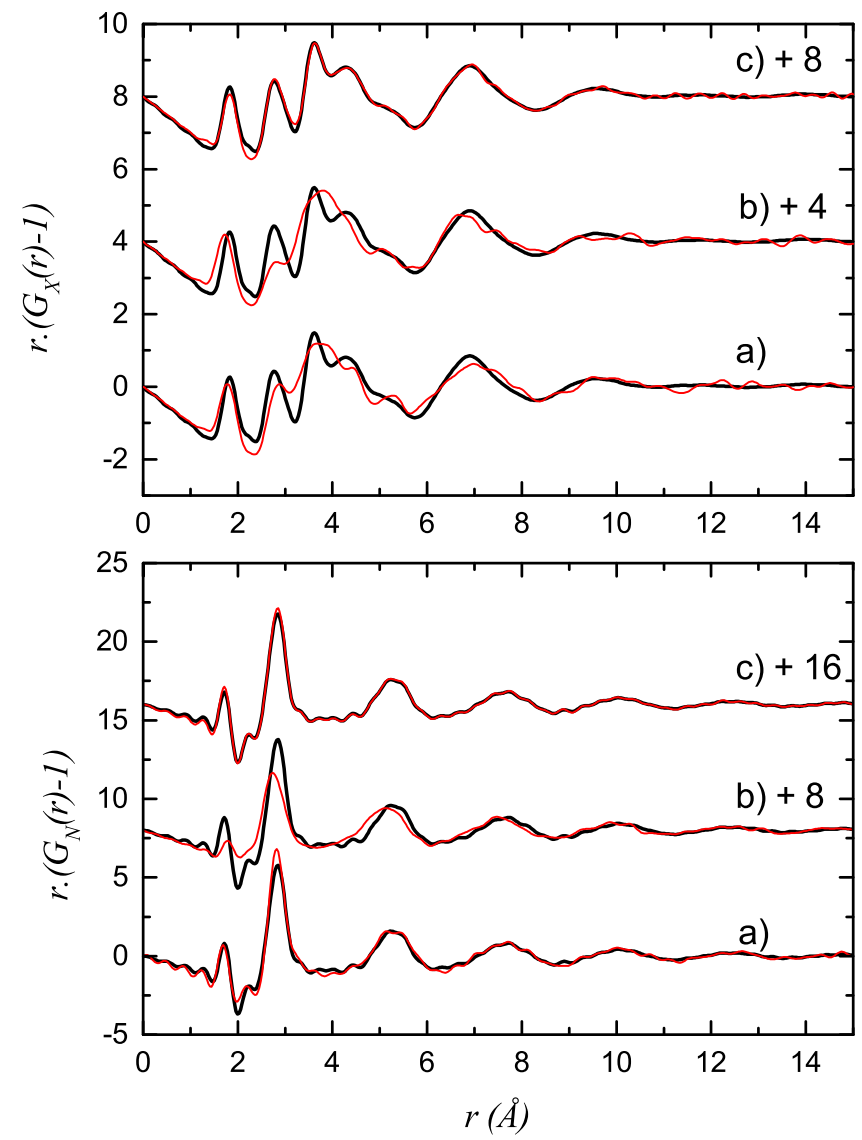

FIG. 5. (Color online) Comparison of the MD and MD-RMC results with the x-ray (upper box) and neutron (lower box) diffraction data (black lines). The data are plotted as $r\left(G_{N / X}(r)-1\right)$ in order to emphasise the fit to the data at high $r$. (a) The MD data (red line) generated with Morse potentials [18] compared to the data. (b) The MD data (red line) generated with Buckingham potentials [17] compared to the data. (c) The MD-RMC fit (red line) to the data. Both (a) and (b) converged to the same MD-RMC result (c) within the resolution of this figure.

There is good agreement between the experimentally measured $Q\left(S_{N}(Q)-1\right)$ and the results calculated from simulation using the Morse potentials of Pedone et al. In contrast, the Buckingham potentials of Bush et al. give a calculated $Q\left(S_{N}(Q)-1\right)$ with poorly matched amplitudes and only weak oscillations at high $Q$.

The corresponding $r\left(G_{N}(r)-1\right)$ function [again shown as $r(G(r)-1)$ to emphasize the high $r$ correlations] is plotted in Fig. 5 and again shows good agreement with the simulation data for the Pedone et al. potentials and poor agreement for the Bush et al. potentials. For the latter, it is clearly seen that the $\mathrm{Al}-\mathrm{O}$ and Ti-O peaks are almost perfectly canceled (due to the negative $\mathrm{Ti}$ scattering length), which explains and is consistent with the lower amplitudes and the lack of high $Q$ oscillations seen in $Q\left(S_{N}(Q)-1\right)$.

For $Q\left(S_{X}(Q)-1\right)$ (Fig. 4), the main features and phase calculated from the simulations are again in broad agreement with the data although there are notable differences in the regions between $\sim 2.5-3.0$ and $\sim 6.0-8.0 \AA^{-1}$ for both sets of potentials. Again, the phasing at high $Q$ for the Bush et al. 
potentials (especially noticeable in the feature at $\sim 7.5 \AA^{-1}$ ) is poorer than observed for Pedone et al. potentials.

The corresponding calculated $r\left(G_{X}(r)-1\right)$ (Fig. 5) again reproduce the broad features well although the peaks in the experimental data are sharper. The first peak calculated from the Bush et al. potentials is at a noticeably shorter distance than observed in the data and, as in the case of the $r\left(G_{N}(r)-1\right)$ suggests that the Bush et al. potentials are underestimating the Ti-O distance in the glass structure.

It is clear from these results that the Pedone et al. potentials give a much better agreement to the data and that this is largely traceable to the shorter Ti-O distance that arises in the case of the potentials from Bush et al. At this point, it is not clear whether the Morse potential is inherently better for simulating these glasses or whether a different set of self consistent Buckingham potentials could be found that would give as good or better agreement. Hence, although our earlier work [11] on Ba-Al-O was based on the Buckingham potentials of Bush et al., the rest of this paper is based on the discussion and refinement of the structure obtained from the Pedone et al. potentials and which is referred to simply now on, as the MD simulation.

The analysis of the MD and RMC configurations was carried out using the ISAACS structure analysis program [47]. A detailed analysis of the MD configuration gives a coordination number of 4.1(1) and 4.4(1) for the Al-O and Ti-O correlations, respectively, when the upper limit of integration is taken as $2.2 \AA$. Analysis of the bond angle distributions (Fig. 10) shows the $\mathrm{O}-\mathrm{Al}-\mathrm{O}$ angle centered at about $105^{\circ}$ (close to the tetrahedral angle of $109^{\circ}$ ) and a broader distribution in the O-Ti-O angle centered around $95^{\circ}$. With a cutoff distance set at $2.2 \AA$, the simulation shows that $90 \%$ of the $\mathrm{Al}$ atoms are fourfold connected to $\mathrm{O}$ with the majority of the remainder (9\%) in fivefold configurations. In contrast, for $\mathrm{Ti}, 60 \%$ of the atoms are fourfold coordinated to $\mathrm{O}$ with the majority of the remainder $(38 \%)$ fivefold coordinated.

It was found that a clear $\mathrm{Ba}-\mathrm{O}$ coordination shell was difficult to observe in the MD configuration. There is a broad minimum in the calculated $g_{\mathrm{BaO}}(r)$ between 3.4 and $3.8 \AA$ with the average $\mathrm{Ba}-\mathrm{O}$ coordination number increasing from $7.9(1)$ to $9.6(1)$ over this range. It was also noted that there is a wide variation in the coordination number of individual $\mathrm{Ba}$ atoms in the configuration such that simple $\mathrm{Ba}-\mathrm{O}$ regular coordination polyhedra with a well defined coordination numbers could not be identified.

\section{RMC refinements}

The RMC procedure used was to run a refinement until a steady $\chi^{2}$ value was obtained for the fit to the data. The procedure was repeated, with different random number seeds, to obtain final configurations that were averaged to calculate the refined $S(Q)$ 's and model parameters (such as coordination number, bond angles, etc.). An example of a final refinement to the x-ray and neutron diffraction data is shown in Fig. 4. The mean displacement of the atoms at the end of each refinement was found to be $0.2 \AA$ with a standard deviation in the distribution of distances of $0.1 \AA$. There was no evidence of preferential movement of any of the atom types and the size of the mean square displacement (i.e., much less than

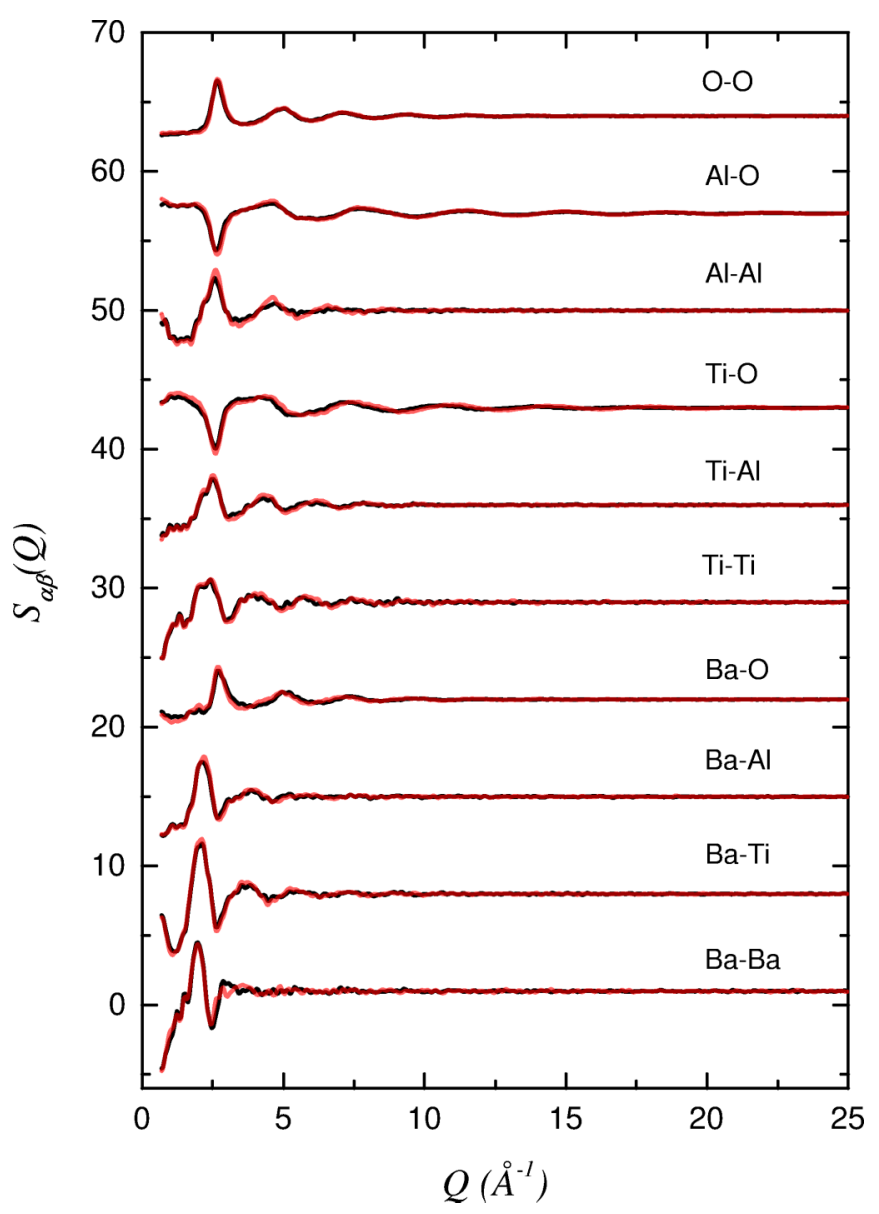

FIG. 6. (Color online) Comparison of the partial structure factors $S_{\alpha \beta}(Q)$ from the MD simulation using Morse potentials [18] (red lines) with those obtained after the MD-RMC refinement (black lines). Each $S_{\alpha \beta}(Q)$ is shifted vertically by 8 .

an interatomic bond distance) confirms this procedure may be considered as a refinement of the initial MD configuration rather than a complete structural reordering.

The $S_{\alpha \beta}(Q)$ and $d_{\alpha \beta}(r) \equiv r\left(g_{\alpha \beta}(r)-1\right)$ from the MD-RMC refinement compared to those obtained from the original MD simulation are shown in Figs. 6 and 7, respectively.

Analysis of the final MD-RMC configurations shows that the position of the first peak in $g_{\mathrm{AlO}}(r)$ is found at 1.73(2) $\AA$ and $g_{\mathrm{TiO}}(r)$ at $1.90(2) \AA$ with evidence for a small low $r$, shoulder at $\sim 1.8 \AA$. Their average coordination numbers reach stable values of $\sim 4$ and $\sim 4.5$, respectively by the position of the first minimum in $G(r)$ at $2.4 \AA$.

The position of the first peak in $g_{\mathrm{BaO}}(r)$ is found to be 2.72(2) $\AA$ and the coordination number is found to vary from 7.4 to 9.3 between distances of 3.4 and $3.8 \AA$, respectively. These values are slightly lower than those observed in the original MD simulation but are very close to those observed in the study of Ba-Al-O by Skinner et al. [11].

\section{X-ray absorption spectroscopy results}

Figure 8(a) shows the XANES spectra obtained around the Ti $K$ edge from the slowly and rapidly quenched BATO samples. In both spectra, there is a notable and significant pre-edge peak at $\sim 4970.4(1) \mathrm{eV}$ followed by the absorption 


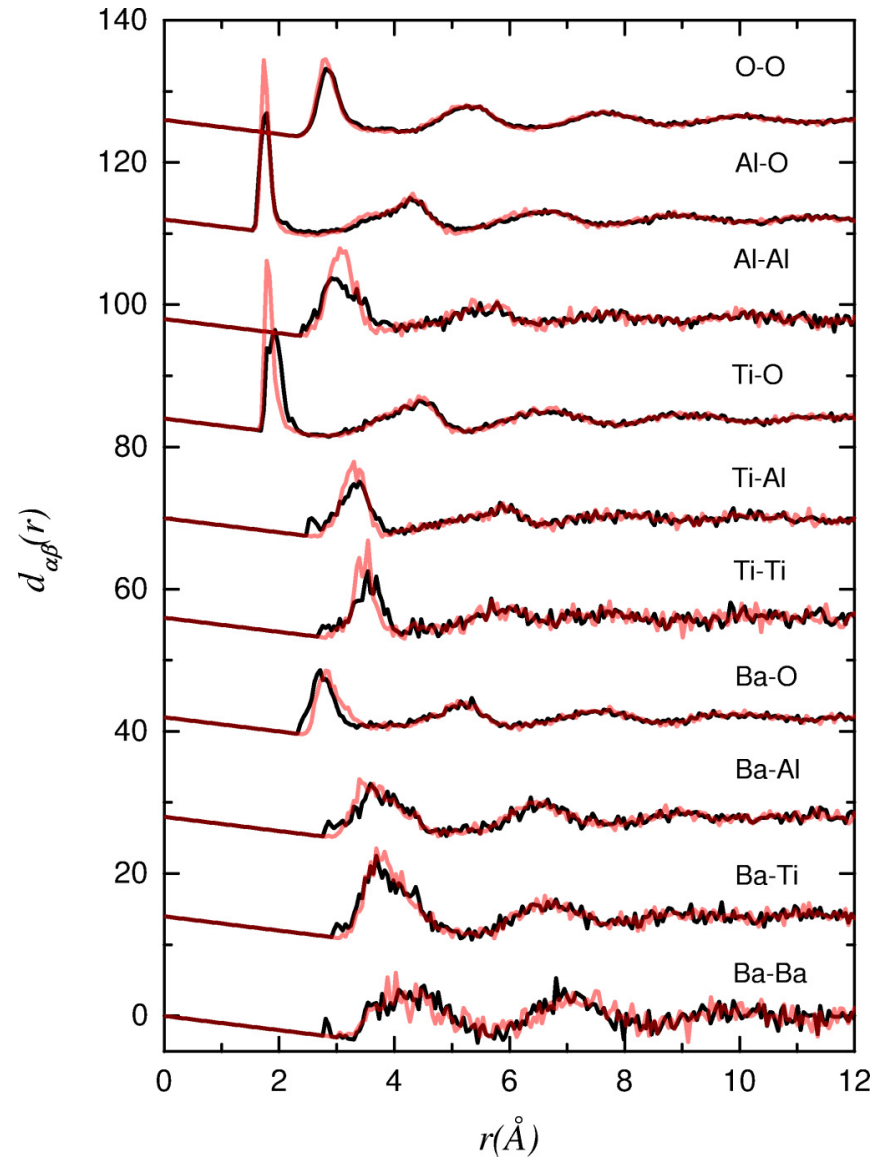

FIG. 7. (Color online) Comparison of pair distribution functions $d_{\alpha \beta}(r) \equiv r\left(g_{\alpha \beta}(r)-1\right)$ from the MD simulation using Morse potentials [18] (red lines) with those obtained after the MD-RMC refinement (black lines). Each $d_{\alpha \beta}(r)$ is shifted vertically by 14 .

edge at $\sim 4982 \mathrm{eV}$. The position of the edge is consistent with the Ti atoms occurring in the $4+$ state. There is a small but noticeable shift in the pre-edge peak to lower energy for the black compared to the clear glass.

The pre-edge feature has been studied extensively in crystals and glasses that contain titanium and it is associated with transitions to the $T_{2 g}$ states occurring due to $p$ - $d$ orbital mixing arising from different structural motifs. The position of the pre-edge peak in this study is consistent with the presence of both fourfold and fivefold coordinated Ti sites that are associated with overlapping peaks at 4969.5 and $4970.5 \mathrm{eV}$, respectively [48]. In the past, quantitative results for the relative numbers of four-, five-, and sixfold coordinated motifs have been calculated by fitting the pre-peak. However, it has been shown recently that the precise shape of this pre-peak is not only dependent on the coordination number but also on the distortion of the structural motifs and the variations in the Ti-O bond distance so that it is difficult to obtain precise values by this method [37,49]. Nevertheless, the Ti XANES structure we observe is consistent with the results of the MD simulations and RMC refinements where predominantly four- and fivefold coordinated $\mathrm{Ti}$ is found.

Figure 8(b) shows the EXAFS spectra extracted for both glasses over the range $2<k<8.5 \AA^{-1}$. The data become unreliable above $k \sim 7.5 \AA^{-1}$ due to an overlap with the Ba

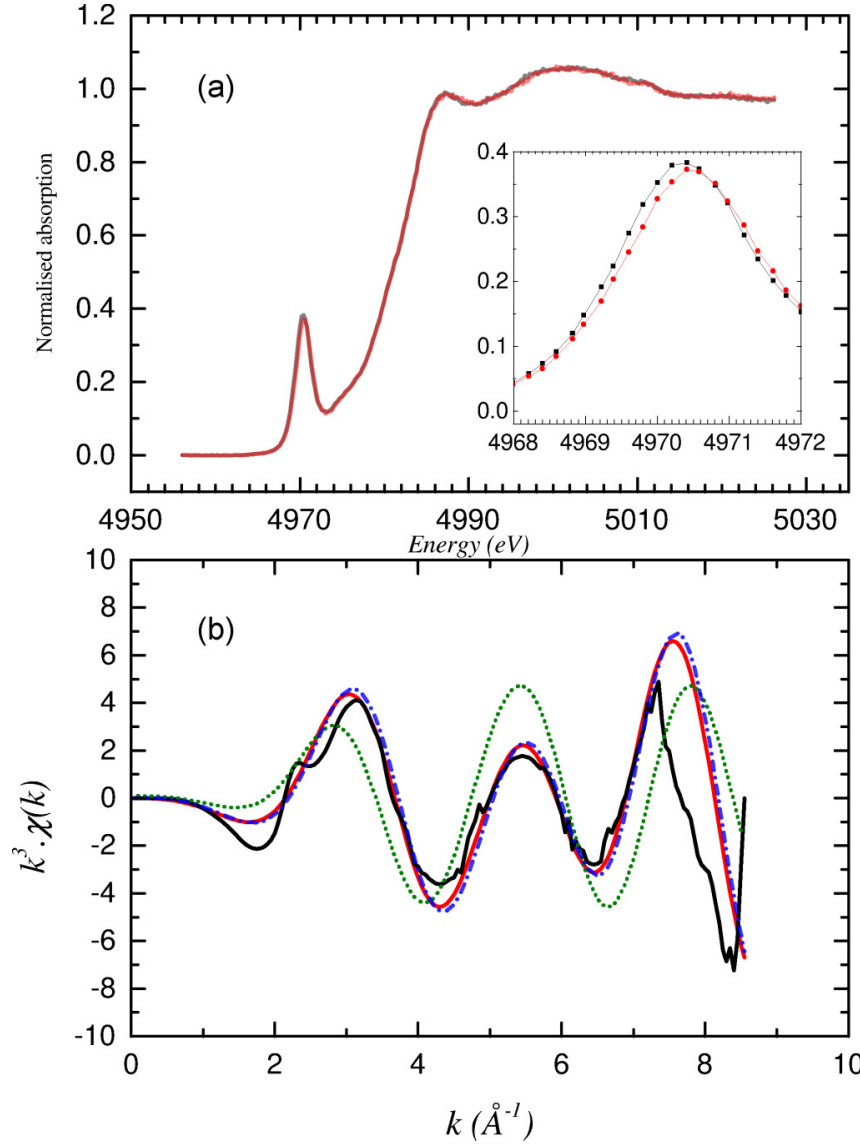

FIG. 8. (Color online) X-ray absorption spectra. (a) The XANES spectra for the black/opaque glass (black) compared to the clear/transparent glass (red-grey). The inset shows the detail of the pre-edge peak in the region $4968-4972 \mathrm{eV}$. (b) The EXAFS spectra for the clear/transparent glass (solid black line) compared with the spectra calculated from the MD configuration using Morse potentials [18] (dot-dash blue line), using Buckingham potentials [17] (dotted green line), and from the final MD-RMC configuration (solid red/grey line).

$L_{3}$ edge at $k \sim 8.5 \AA^{-1}(5.25 \mathrm{keV})$. This leaves a k-range that is insufficient to carry out reliably, either a Fourier transform of the data, or a direct fit to the spectra as is usually applied to EXAFS data. In addition, the MD-RMC analysis strongly suggests a minimum of two different Ti coordination sites in the glass (fourfold and fivefold) so that it is difficult to choose a suitable cluster configuration for a conventional EXAFS refinement using, for example, FEFFIT [35]. In contrast, the MD and MDRMC configurations allow a site by site calculation of the EXAFS spectra for each Ti atom (for example, using FEFF [34]) so that an average EXAFS spectrum for all atom clusters in these configurations can be compared with the measured spectra.

Individual Ti clusters were extracted from the MD and MDRMC configurations with the cutoff distance determined by the observed minimum in $g_{\mathrm{TiO}}(r)$ at $2.3 \AA$. The EXAFS signal was calculated for each cluster separately and the final result for the whole sample was obtained from the average value over all these clusters. The value of $S_{0}^{2}$ and the Debye-Waller factor were set to 1 and 0 , respectively, as the disorder was naturally incorporated in the averaging over all the clusters in 
the configurations. For similar reasons, we did not attempt to include higher cumulant terms in the calculated spectra.

Figure 8(b) shows the calculation of the averaged EXAFS signals for all $\mathrm{Ti}$ sites for the MD configurations generated from both the Buckingham potentials [17] and Morse potentials [18] and the MD-RMC configuration obtained from the MD simulation with Morse potentials. There is very poor agreement in both phase and amplitude with the MD simulation using the Buckingham potentials. This confirms the poor ability of these potentials to account for the titanium bond distance and coordination. In contrast, the agreement between the MD configuration from the Morse potentials and the final MD-RMC is good and supports the conclusion that the local Ti coordination is reproduced well using this method.

\section{DISCUSSION}

\section{A. The coordination of $\mathrm{Al}$ and $\mathrm{Ti}$ atoms in the glass}

It is interesting to compare the results of this study with the results reported by Skinner et al. [11] with respect to pure $\mathrm{BaAl}_{2} \mathrm{O}_{4}$ glass (which we henceforth refer to as $\mathrm{BAO}$ glass in this discussion). In BAO glass, the Al-O coordination was found to be broadly tetrahedral despite an $\mathrm{O}$ :Al ratio of 1.8 , which is less than the ratio needed to support a fully coordinated corner shared tetrahedral glass network. The analysis and conclusion of the work was that there were a significant number of oxygen "triclusters" in which some oxygen atoms are threefold coordinated to $\mathrm{Al}$ rather than the complete twofold coordination expected in a perfect tetrahedral network. In the BATO glass studied here, we consider that the $\mathrm{Al}$ and $\mathrm{Ti}$ atoms both participate in the connected oxide network structure. Hence it is instructive to note that the $(\mathrm{Al} / \mathrm{Ti}): \mathrm{O}$ ratio in these glasses is very close to 2 , the value needed to form a perfectly tetrahedrally corner shared network without the need to form either $\mathrm{Al}$ or $\mathrm{Ti}$ triclusters. However, the results from the MD simulation and the MD-RMC refinement suggest that this is not the oxide network structure that is adopted. As observed previously, due to the overlap of the Al-O and Ti-O peaks in the total $G_{N / X}(r)$ it is not possible to obtain coordination number by direct integration of the real-space data. However, details of the coordination structures around these atoms maybe obtained directly from the MD and MD-RMC configurations.

Figure 9(a) shows the running coordination number

$$
n(r)=4 \pi \rho_{N} \int_{0}^{r} r^{\prime 2} g\left(r^{\prime}\right) d r^{\prime},
$$

for Ti-O and Al-O correlations in the glass obtained from the MD-RMC configuration. The Al-O coordination number reaches a value of 4 at $\sim 2.4 \AA$ [the first minimum in $g_{\mathrm{AlO}}(r)$ ]. Figure 9(b) shows the distribution of the $n$-fold $(n=2,3,4$, 5 , or 6 ) coordinated $\mathrm{Al}$ sites as a function of $r$ over the same range. From this figure it can be seen that the average Al-O coordination number is due almost entirely (90\%) to fourfold coordinated sites with small numbers of three- and fivefold coordinated sites. Figure 10(a) shows the O-Al-O bond angle distribution obtained using an Al-bond distance cutoff distance of $2.4 \AA$ from both the MD and MD-RMC configurations. The mean bond angle is centered around $104^{\circ}$; it is largely unchanged after the refinement and suggests further that the
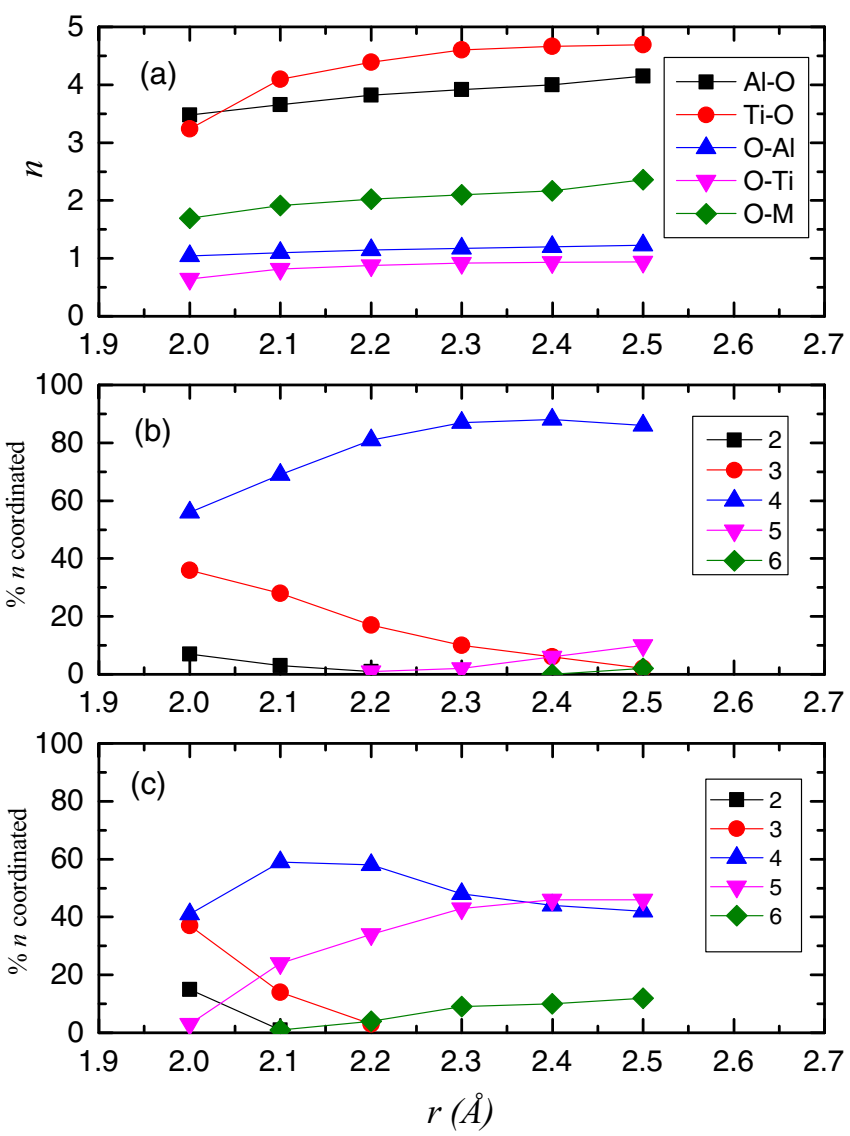

FIG. 9. (Color online) Coordination numbers. (a) The coordination number $n$ for $\mathrm{O}$ around $\mathrm{Al}$ (black squares), $\mathrm{O}$ around Ti (red circles), $\mathrm{Al}$ around $\mathrm{O}$ (blue triangles), $\mathrm{O}$ around $\mathrm{Ti}$ (inverted magenta triangles), and $\mathrm{M}$ around $\mathrm{O}$ (green diamonds), where $\mathrm{M}$ is either Ti or Al. (b) Percentage of $n$-fold $\mathrm{O}$ coordinated $\mathrm{Al}$ atoms as a function of distance. (c) Percentage of $n$-fold $\mathrm{O}$ coordinated Ti atoms as a function of distance.

Al-O coordination is tetrahedral in nature. These conclusions are similar to those obtained in the study of BAO glass and suggests that the local Al-O order is largely unaffected by the addition of $\mathrm{Ti}$.

Moving to the Ti-O coordination it is seen in Fig. 9(a) that the average Ti-O coordination number reaches a value of $\sim 4.5$ at $2.4 \AA$. Figure 9 (c) shows the distribution of $n$-fold ( $n=2,3,4,5$, or 6 ) coordinated sites as a function of $r$ over the same range. From this result, it is seen that the average coordination number arises from almost equal numbers of four- and fivefold coordinated sites but with a small $(\sim 10 \%)$ but significant number of sixfold coordinated sites. Figure 10(b) shows the O-Ti-O bond angle distribution obtained from both the MD and MD-RMC configurations. Unlike the O-Al-O distribution, there is a clear change from the MD configuration to the MD-RMC configuration, most notably a considerable weakening of the peak at $>100^{\circ}$ and strengthening of the peak at $\sim 90^{\circ}$. In addition there is a notable rise in the distribution at angles close to $180^{\circ}$.

The local coordination geometry of oxygen around titanium in Ti compounds is well known to give strong features in the pre-edge region of the x-ray absorption spectra. In particular, in crystals, tetrahedral $\mathrm{TiO}_{4}$ units give a very strong and narrow 


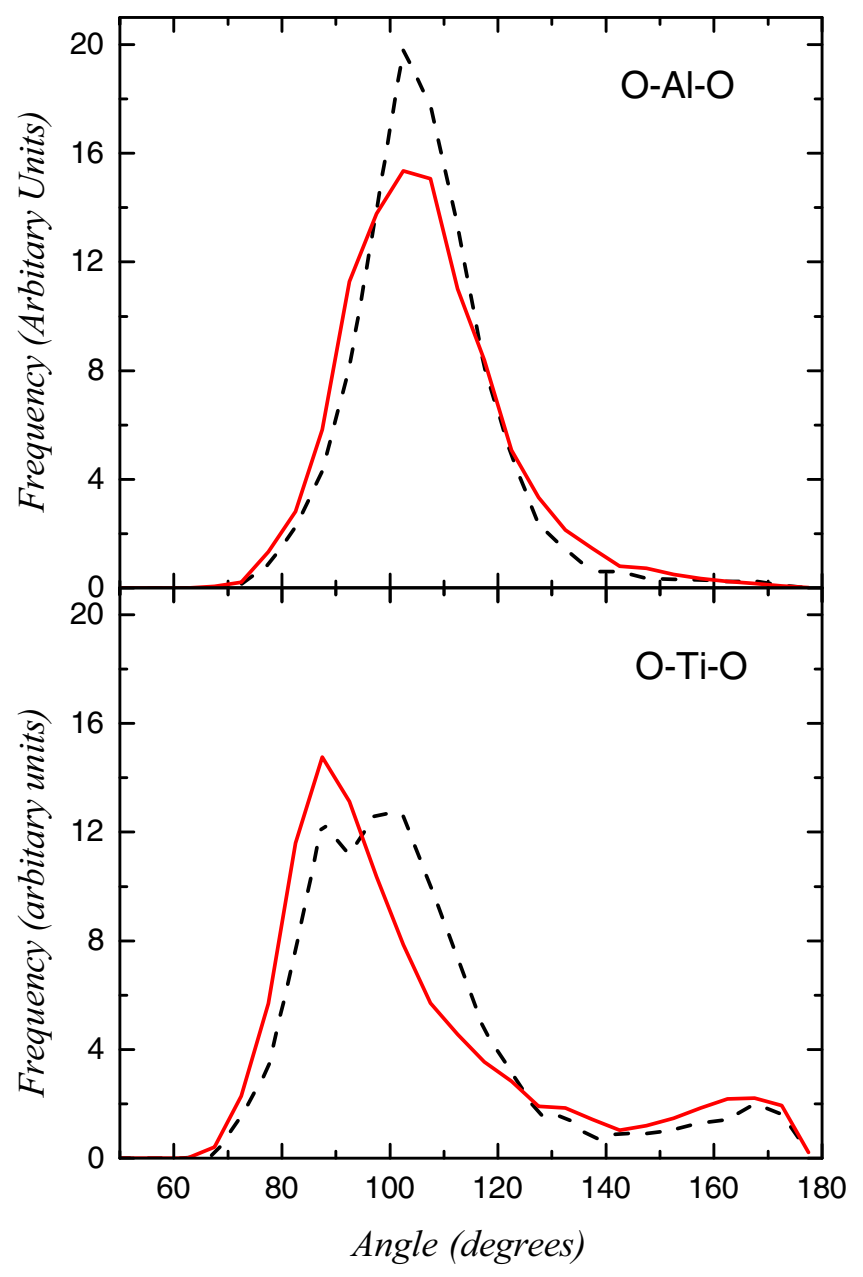

FIG. 10. (Color online) O-Al-O (top) and O-Ti-O (bottom) bond angles obtained from the MD simulation with Morse potentials [18] (black dashed line) and after the MD-RMC refinement (red solid line).

peak centered at $4969.5 \mathrm{eV}$ whereas fivefold structural motifs give rise to a peak with about half this height at $4970.5 \mathrm{eV}$ [48]. Hence the peak observed in our XANES spectra at $\sim 4970.4(1) \mathrm{eV}$ (Fig. 8) is consistent with the overlap of peaks originating from the four- and fivefold coordinated structures in the MD-RMC configuration. However, as noted by Tamura et al. [49] and Jiang et al. [37], the conventional fingerprint approach of fitting crystalline peak positions and heights for four-, five- and sixfold spectra [48] to the pre-edge feature in glassy systems is limited due to the complexity and variation in the local $\mathrm{Ti}$ environment. Indeed, there is still some debate concerning the precise electronic origin of pre-edge features in Ti XANES spectra $[37,49]$. However, as noted above the XANES pre-peak for tetrahedrally coordinated $\mathrm{TiO}_{4}$ units is narrow and strong (typically comparable to the Ti $K$-edge jump) and hence might still be expected to dominate the pre-edge feature. However, this is not seen in the data and broadly supports the conclusion from the MD-RMC analysis that the fourfold coordinated Ti sites are not strongly tetrahedrally coordinated.

The neutron and $\mathrm{x}$-ray diffraction patterns for the clear and black glasses are statistically identical such that no discernible difference between them could be identified. However, the Ti XANES spectra do show a small difference with a slight shift to lower energy for the pre-edge feature for the black (rapidly quenched) glass [see the inset in Fig. 8(a)]. This is the only difference we have found between the two samples in this study apart from their color. XANES, as a spectroscopic technique, is very sensitive to subtle changes in the Ti coordination and this shift suggests a slightly higher tendency for fourfold and perhaps tetrahedral coordination to be present in the black glass. A change in the position of the absorption edge, that would correspond to a major change in the Ti oxidation state, is not observed. The MD simulation, that was equilibrated in a high-temperature liquid state and quenched at a physically unrealistic time scale to room temperature, showed a considerably higher number of tetrahedrally coordinated Ti than the final MD-RMC refinement. However, discoloration due to a change in the Ti coordination appears unlikely, irrespective of whether there are a fractionally higher number of tetrahedrally connected Ti atoms. It therefore remains most likely that the discoloration is due to a very low density of reduced $\mathrm{Ti}\left(\mathrm{Ti}^{3+}\right.$ or $\mathrm{Ti}^{2+}$ ) sites in the glass as suggested by Jijian and Wei [12].

The average $\mathrm{O}-M$ coordination number $(M=\mathrm{Al}$ or Ti) is calculated as 2.1, which is consistent with significant numbers of oxygen triclusters in the network structure as also found in BAO glasses [11]. A detailed examination of the MD-RMC configuration file shows that approximately $67 \%$ of the oxygen atoms in the structure are twofold coordinated, 20\% threefold coordinated, and the remaining mostly singly coordinated to $\mathrm{Al}$ and $\mathrm{Ti}$ atoms. Nevertheless, the low oxygen-metal coordination number still leads to a general picture of the network glass structure arising from highly connected $\mathrm{M}: \mathrm{O}$ motifs interconnected by oxygen atoms with low coordination number.

\section{B. The network glass structure}

It is interesting to compare how the different short-range order around the $\mathrm{Al}$ and $\mathrm{Ti}$ atoms in this glass affects the nature of the oxide glass network structure, especially in comparison to the archetypal tetrahedral glasses such as $\mathrm{SiO}_{2}$ and $\mathrm{GeO}_{2}$ [50] and pure $\mathrm{Ba}-\mathrm{Al}-\mathrm{O}[11]$ glass where the structure is usually discussed in terms of the packing of regular tetrahedral motifs. Figures 6 and 7 show the partial structure factors and partial pair distribution functions obtained from the MD and MDRMC configurations, respectively. It is noticeable that there is essentially no difference between the partial $d_{\alpha \beta}(r)$ between the MD configuration and the final MD-RMC refinement above $\sim 4 \AA$. Most strikingly, it is seen that $d_{\mathrm{OO}}(r)$ is virtually unchanged over all length scales. It hence appears that the medium to long-range order in the glass is reproduced well by the simple pair potentials used in the simulation and that it is the competition between the long-range order and the strength of the local topological bonding requirements that characterises the oxide glass network structure. For glasses with structures based purely on the packing of regular tetrahedral units, the relative strength of the long range, compared to the local order, is given as an explanation for the differences in their fragility and the strength of the characteristic first sharp diffraction peak (FSDP) observed in their diffraction patterns [50]. In Ba-Al-O, a weak FSDP is found in the network structure that is consistent with it being a relatively fragile glass formed by the packing 


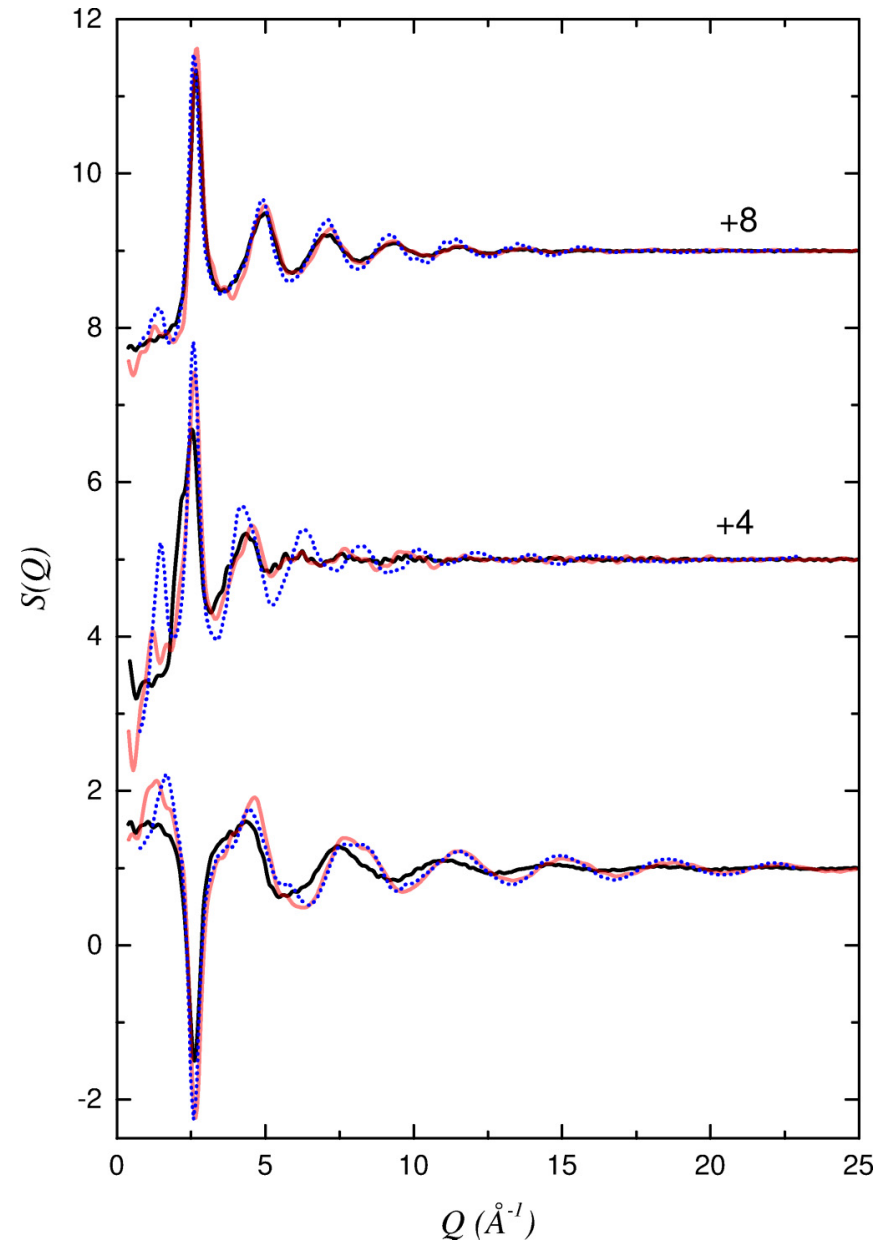

FIG. 11. (Color online) The $S_{\mathrm{OO}}(Q)$ (top), $S_{M M}(Q)$ (middle), and $S_{M O}(Q)$ (bottom), where $M=\mathrm{Al}$ or Ti Faber-Ziman partial structure factors (black line) when compared with their analogues in $\mathrm{BaAl}_{2} \mathrm{O}_{4}(M=\mathrm{Al})$ [solid red (grey) line] and $\mathrm{GeO}_{2}(M=\mathrm{Ge})$ (dotted blue lines)glass.

of $\mathrm{AlO}_{4}$ tetrahedra in which the local ordering is weakened by the formation of a significant fraction of tetrahedra connected by oxygen triclusters.

It is clear in BATO glass that the restrictions imposed by the ordering of regular tetrahedra no longer apply and that the ability of $\mathrm{Ti}$ to form differently coordinated motifs relaxes considerably the constraints imposed by the short-range order in forming the glass.

A clearer picture of the topology and order in the oxide network in BATO maybe obtained by examining the structure of the $M-\mathrm{O}$ network where $M=\mathrm{Al}$ or Ti. The atomic number density of the atoms in this network is $0.064 \AA^{-3}$ which is, for comparison $0.061 \AA^{-3}$ in BAO and $0.0629 \AA^{-3}$ in $\mathrm{GeO}_{2}$ glass. Figure 11 shows a comparison of the Faber-Ziman partial structure factors $S_{M M}(Q), S_{M \mathrm{O}}(Q)$, and $S_{\mathrm{OO}}(Q)$ obtained from the MD-RMC compared to the $\mathrm{Al} / \mathrm{O}$ and $\mathrm{Ge} / \mathrm{O}$ partial structure factors of $\mathrm{BAO}$ [11] and $\mathrm{GeO}_{2}$ [50] glass. Most striking is the very close similarity of $S_{\mathrm{OO}}$ in these glasses where the only difference in BATO is the absence of any FSDP [51] feature at $\sim 1 \AA^{-1}$ and the more heavily damped oscillations.

For $S_{M M}(Q)$, BATO and BAO are very similar, apart again, from the absence of any FSDP in BATO, whereas for $\mathrm{GeO}_{2}$,

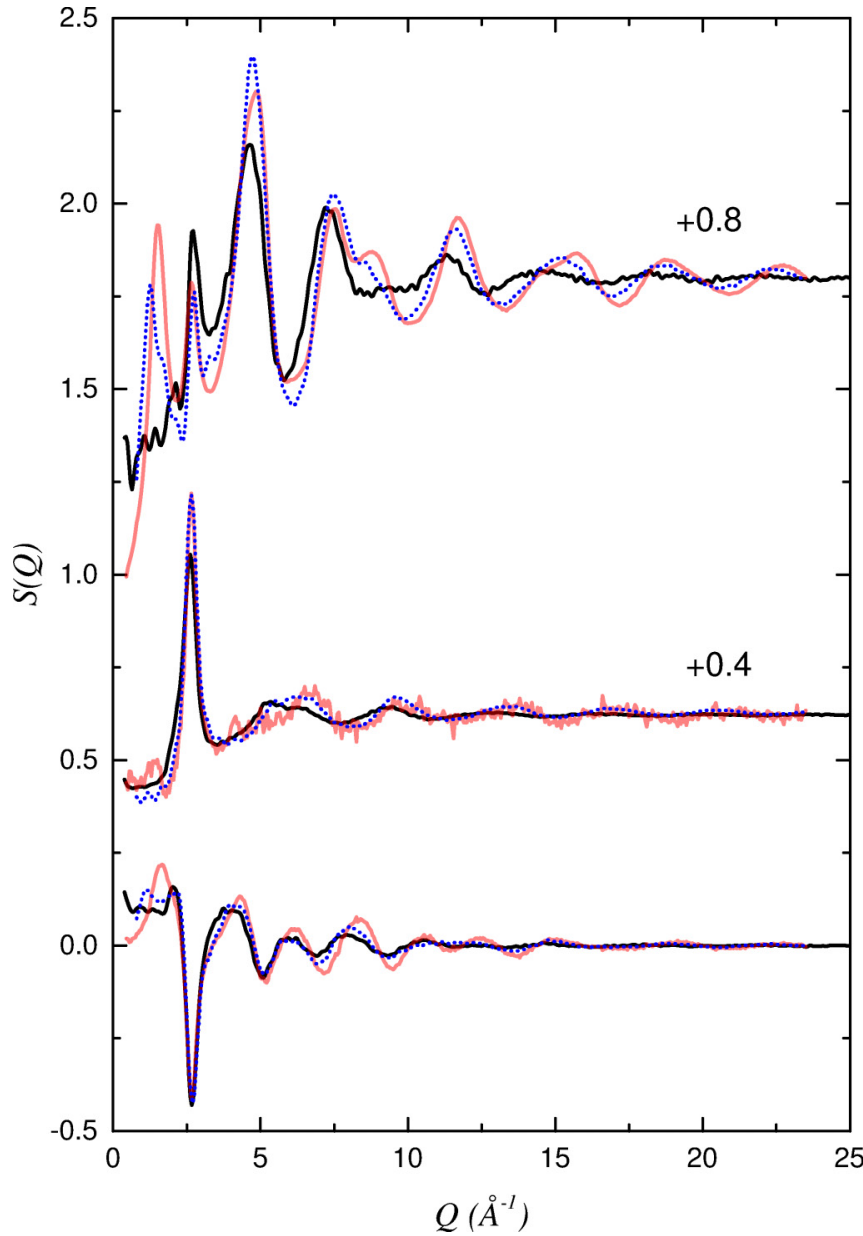

FIG. 12. (Color online) The $\mathrm{M}-\mathrm{O}$ (where $M=\mathrm{Al}$ or Ti) network partial structure factors $S_{N N}(Q)$ (top), $S_{C C}(Q)$ (middle), and $S_{N C}(Q)$ in the Bhatia-Thornton representation (solid black lines) when compared with their analogues in $\mathrm{BaAl}_{2} \mathrm{O}_{4}(M=\mathrm{Al})$ [solid red (grey) line] and $\mathrm{GeO}_{2}(M=\mathrm{Ge})$ (dotted blue lines) glass.

the FSDP is very strong and the amplitude of the oscillations is much stronger. From the differences observed it appears that as the constraints on the $M-O$ motifs are relaxed, through triclustering in BAO and in addition the presence of different and more relaxed Ti-O polyhedra types in BATO, the $M$ atoms are able to take up much less rigidly constrained positions in the oxide network.

The differences in $S_{M O}(Q)$ are different. In this case, the close similarity in $\mathrm{GeO}_{2}$ and $\mathrm{BAO}$ reflects the uniformity and rigidity of the characteristic tetrahedral motifs. In comparison, the difference in the Ti-O and Al-O bond distances and the additional presence of five- and sixfold coordinated Ti shifts the phase and the damping of the oscillations and is consistent with reduction in rigidity and the increasing number of the types of structural motif in BATO.

An alternative viewpoint of the $M-\mathrm{O}$ network structure is to compare and contrast the pseudobinary Bhatia-Thornton structure factors $S_{N N}(Q), S_{N C}(Q)$, and $S_{C C}(Q)$ [52] as described in the discussion of BAO [11] and shown in Fig. 12. $S_{N N}(Q)$ represents the topological (independent of atom type) ordering in the glass and emphasises the differences in the structure of the network. Strong tetrahedral glasses such as 
$\mathrm{GeO}_{2}$ show a prominent FSDP and a characteristic pattern of oscillations at high $Q$. The height and strength of the FSDP in $S_{N N}(Q)$ is often associated with the fragility [53] of the glass. In BAO, $S_{N N}(Q)$ showed strong similarities, including a smaller but significant FSDP, to that observed in $\mathrm{GeO}_{2}$. However, in BATO there is no evidence of any FSDP, and the high- $Q$ region is markedly different. This is perhaps, at first sight, surprising given that $S_{\mathrm{OO}}(Q)$ is so similar in all of these glasses. However, it strongly reflects the notably different organization of the metal ions in the network that arise due to the presence of the multiply coordinated Ti centers.

The absence of an FSDP in any of the network partial structure factors in BATO is interesting. The position, height and sharpness of this peak is often associated with the fragility of a glass; the more prominent the feature is, the stronger the glass and its glass forming ability is. In 1:2 materials such as $\mathrm{SiO}_{2}, \mathrm{GeO}_{2}, \mathrm{GeS}_{2}, \mathrm{GeSe}_{2}$, the FSDP is closely related to the packing and organization of the basic $M X_{4}$ structural units. Hence with this picture, the complete absence of evidence for a FSDP in BATO suggests that it should be extremely fragile and a very poor glass former. However, it readily forms a glass on our levitation system and its glass forming abilities are similar to that of BAO. Although our results show that in BATO, Al is almost universally tetrahedrally coordinated (as in BAO), it appears that the significant number of fivefold and irregularly fourfold coordinated $\mathrm{Ti}$ atoms in the glass network are sufficient to relax the constraints for packing the regular $\mathrm{AlO}_{4}$ tetrahedra. Hence, we could, from this study conclude that the presence of an FSDP is not a necessary condition for oxide materials to be a moderately good glass former.

$S_{C C}(Q)$ and $S_{N C}(Q)$ are strongly associated with the chemical ordering in the material. In this regard, it is notable how $S_{C C}(Q)$ is very similar in BAO and BATO but different to that observed in $\mathrm{GeO}_{2}$. It is notable that the biggest differences over this $Q$ range are observed in $S_{N N}(Q)$ where the correlations from the strong uniformly tetrahedrally coordinated structure of for example $\mathrm{GeO}_{2}$ have largely disappeared. The large number of nontetrahedrally coordinated $\mathrm{Ti}-\mathrm{O}$ motifs in this glass have a dramatic effect on the medium-range order (MRO) where the FSDP as all but disappeared.

The x-ray and neutron diffraction patterns both show a rise in the scattering at small $Q$ giving evidence of some homogeneities in the glass over relatively short distances. Similar effects were not seen in BAO glass and it is possible this rise could be associated with some ordering of $\mathrm{Al}$ and $\mathrm{Ti}$ over these length scales. Interestingly, when at the glass forming threshold, BATO forms turbid glassy material. A tendency for nanoscale phase separation as evidenced by this rise in scattering may be an explanation for this behavior and would be interesting to explore by small angle $\mathrm{x}$-ray or neutron scattering.

\section{The Ba coordination structure}

Up until this point, we have not discussed the role of the barium atoms in the glass. Its primary role in this glass is to charge balance the extra oxygen introduced into the glass network to bring the $(\mathrm{Al} / \mathrm{Ti}): \mathrm{O}$ ratio close to 2 . It is not considered to form any strong directional covalent bonds that are directly coordinated to the oxygen in the network. It can be noted in Fig. 7 that the Ba correlations remain largely unchanged in the MD-RMC refinement apart from a slight broadening and small shift to low $r$ in the nearest neighbor Ba-O peak. In addition, the first minimum in $d_{\mathrm{BaO}}(r)$ is weak and poorly defined suggesting that there are no strongly defined $\mathrm{BaO}$ structural motifs in the glass. This is also reflected in the determination of the $\mathrm{Ba}-\mathrm{O}$ coordination number that shows an approximately linear relationship between 5.6 and 9.4 over the range $3.0<r<3.8 \AA$. The $\mathrm{Ba}-\mathrm{O}$ coordination number observed in this glass compared with that of BAO at a cut-off distance of $3.4 \AA$ is identical (within error) with a value of 7.4 and very close at a 9.3 compared to 9.4 at a cutoff distance of $3.8 \AA$. It thus appears that the $\mathrm{Ba}-\mathrm{O}$ coordination structure in these glasses is closely related and unaffected by the changes in the network structure caused by the addition of Ti as discussed above.

Hollandite type Ba-Al-Ti-O crystalline materials (SYNROC) have been proposed for the encapsulation of nuclear waste, especially with regard to the large $\mathrm{Cs}^{+}$ion $[3,10]$. The ability of BATO glass to accommodate the large $\mathrm{Ba}^{2+}$ ion suggests that it should be possible to encapsulate $\mathrm{Cs}^{+}$in the glass making it of interest for vitreous waste encapsulation.

\section{Comments on the potentials used in the MD simulations}

In this study, we have carried out a simulation of the glass using simple pair potentials from the literature. These were the Buckingham potentials of Bush et al. [17] and Morse potentials of Pedone et al. [18]. Although the potentials of Bush et al. were used successfully to simulate and refine the structure of Ba-Al-O glass by Skinner et al. [11] it was found that inclusion of the Ti potentials from the same study gave a poor agreement with the data for the Ba-Al-Ti-O glass studied here. In contrast, the potentials from Pedone et al. gave excellent agreement with the data, especially with $S_{N}(Q)$. The agreement is much poorer with $S_{X}(Q)$ and is related to the heavier weighting of the $\mathrm{Ba}$ partial structure factors. This suggests that there is still scope for improving, especially, the $\mathrm{Ba}-\mathrm{O}$ potential in the MD simulations. Nevertheless, we believe the initial close agreement of the MD results using the Morse potentials and the small atom displacements necessary to refine the configuration to the experimental data show that this method is a good approach to obtain an atomistic model of this four component glass where it is not possible, experimentally, to isolate partial structure factors.

\section{CONCLUSIONS}

The structure of $\mathrm{Ba}_{0.09} \mathrm{Al}_{0.18} \mathrm{Ti}_{0.12} \mathrm{O}_{0.61}$ glass was investigated by neutron and $\mathrm{x}$-ray diffraction, XAS and MD simulation. The basic structure of the glass was produced well from an MD simulation using Morse type pair potentials. An RMC refinement of the MD atomic structure of this glass reveals close similarities to that found previously in Ba-Al-O glass. In both of these glasses, the $\mathrm{Ba}-\mathrm{O}$ coordination remains largely unchanged without any clear requirement for a regular $\mathrm{Ba}-\mathrm{O}$ structural motif. A close examination of the network $(\mathrm{Al} / \mathrm{Ti}-\mathrm{O})$ structure reveals that the $\mathrm{Al}$ coordination remains dominated by regular tetrahedral $\mathrm{AlO}_{4}$ motifs whereas the Ti coordination is characterised by four- and fivefold coordinated 
sites with less well defined structural motifs. The structure of the black/opaque and clear/transparent glasses is identical from a diffraction perspective but Ti XANES spectra show a small tendency towards more regular tetrahedral motifs in the black rapidly quenched glasses. However, we still conclude that the most likely reason for the discoloration is due to very small numbers of reduced valence $\mathrm{Ti}$ sites. The presence of four- and fivefold $\mathrm{Ti}$ sites in the network structure leads to the absence of notable medium range order as evidenced by the lack of a first sharp diffraction peak. However, despite the absence of an FSDP, this material is still able to form a good glass at similar quench rates to $\mathrm{Ba}-\mathrm{Al}-\mathrm{O}$ glass.

\section{ACKNOWLEDGMENTS}

P. Kidkhunthod would like to acknowledge the Royal Thai government for the award of a Ph.D. scholarship. A. Barnes would like to acknowledge the support of the EPSRC (Grant EP/F021488/1). This work was supported by the US Department of energy, office of basic energy sciences, under Grant No. DE-FG02-09ER46650 (x-ray experiment and manuscript preparation, LBS), and contract DE-AC0206CH11357 (Argonne National Lab.). We would like to thank P. Palleau (ILL) for his assistance with the neutron diffraction experiments.
[1] A. E. Ringwood, S. E. Kesson, N. G. Ware, W.Hibberson, and A. Major, Nature (London) 278, 219 (1979).

[2] F. P. F. Van Berkel, H. W. Zandbergen, G. C. Verschoor, and D. J. W. Ijdo, Acta Cryst. C 40, 1124 (1984).

[3] J.-M. Montel, Comptes Rendus Geoscience 343, 230 (2011).

[4] K. Trachenko, E. Zarkadoula, I. Todorov, M. Dove, D. Dunstan, and K. Nordlund, Nucl. Instrum. Methods Phys. Res., Sect. B 277, 6 (2012).

[5] J. Yu, S. Kohara, K. Itoh, S. Nozawa, S. Miyoshi, Y. Arai, A. Masuno, H. Taniguchi, M. Itoh, M. Takata, T. Fukunaga, S.-y. Koshihara, Y. Kuroiwa, and S. Yoda, Chem. Mater. 21, 259 (2009).

[6] G. Gao, S. Reibstein, E. Spiecker, M. Peng, and L. Wondraczek, J. Mater. Chem. 22, 2582 (2012).

[7] M. Allix, S. Alahrache, F. Fayon, M. Suchomel, F. Porcher, T. Cardinal, and G. Matzen, Adv. Mater. 24, 5570 (2012).

[8] X. Wang, Y. Zhang, L. Cui, and I. S. Baturin, Ferroelectrics 442, 109 (2013).

[9] Y. Zhang, A. Navrotsky, J. A. Tangeman, and J. K. R. Weber, J. Phys.: Condens. Matter 15, S2343 (2003).

[10] L. B. Skinner, A. C. Barnes, and W. Crichton, J. Phys. Condens. Matter 18, L407 (2006).

[11] L. B. Skinner, A. C. Barnes, P. S. Salmon, H. E. Fischer, J. W. E. Drewitt, and V. Honkimäki, Phys. Rev. B 85, 064201 (2012).

[12] C. Jijian and C. Wei, J. Non-Cryst. Solids 80, 135 (1986).

[13] L. B. Skinner, A. C. Barnes, P. S. Salmon, and W. A. Crichton, J. Phys. Condens. Matter 20, 205103 (2008).

[14] S. Aasland and P. McMillan, Nature (London) 369, 633 (1994).

[15] G. N. Greaves, M. C. Wilding, S. Fearn, D. Langstaff, F. Kargl, S. Cox, Q. V. Van, O. Majrus, C. J. Benmore, R. Weber, C. M. Martin, and L. Hennet, Science 322, 566 (2008).

[16] A. C. Barnes, L. B. Skinner, P. S. Salmon, A. Bytchkov, I. Pozdnyakova, T. O. Farmer, and H. E. Fischer, Phys. Rev. Lett. 103, 225702 (2009).

[17] T. S. Bush, J. D. Gale, C. R. A. Catlow, and P. D. Battle, J. Mater. Chem. 4, 831 (1994).

[18] A. Pedone, G. Malavasi, M. C. Menziani, A. N. Cormack, and U. Segre, J. Phys. Chem. B 110, 11780 (2006).

[19] H. E. Fischer, A. C. Barnes, and P. S. Salmon, Rep. Prog. Phys. 69, 233 (2006).

[20] H. Rauch and W. Waschkowksi, Neutron Data Booklet, 2nd ed. (Old City Publishing, Philadelphia, 2003), Chap. 1.1.

[21] E. N. Maslen, A. G. Fox, and M. A. O'Keefe, International Tables for Crystallography (Kluwer Academic, Dordrecht, 1995), Chap. 6.1.1, p. 476.
[22] D. T. Cromer, J. Appl. Crystallogr. 16, 437 (1983).

[23] R. Car and M. Parrinello, Phys. Rev. Lett. 55, 2471 (1985).

[24] D. Marx and J. Hutter, in Modern Methods and Algorithms of Quantum Chemistry, Vol. NIC series 3, edited by J. Grotendorst (John Von Neumann Institute for Computing, Julich, 2000), p. 329.

[25] M. P. Allen and D. J. Tildesley, Computer Simulation of Liquids (Oxford University Press, Oxford, 1989).

[26] C. A. Angell and L. M. Torell, J. Chem. Phys. 78, 937 (1983).

[27] T. F. Soules, J. Non-Cryst. Solids 49, 29 (1982).

[28] R. A. Buckingham, Proc. R. Soc. London, Ser. A 168, 264 (1938).

[29] P. M. Morse, Phys. Rev. 34, 57 (1929).

[30] L. B. Skinner, A. C. Barnes, P. S. Salmon, L. Hennet, H. E. Fischer, C. J. Benmore, S. Kohara, J. K. Richard Weber, A. Bytchkov, M. C. Wilding, J. B. Parise, T. O. Farmer, I. Pozdnyakova, S. K. Tumber, and K. Ohara, Phys. Rev. B 87, 024201 (2013).

[31] R. L. McGreevy and L. Pusztai, Mol. Simul. 1, 359 (1988).

[32] M. G. Tucker, D. A. Keen, M. T. Dove, A. L. Goodwin, and Q. Hui, J. Phys. Condens. Matter 19, 335218 (2007).

[33] J. J. Rehr and R. C. Albers, Rev. Mod. Phys. 72, 621 (2000).

[34] A. L. Ankudinov, B. Ravel, J. J. Rehr, and S. D. Conradson, Phys. Rev. B 58, 7565 (1998).

[35] M. Newville, B. Ravel, D. Haskel, J. Rehr, E. Stern, and Y. Yacoby, Physica B: Condensed Matter 208-209, 154 (1995).

[36] A. Filipponi, J. Phys. Condens. Matter 13, R23 (2001).

[37] N. Jiang, D. Su, and J. C. H. Spence, Phys. Rev. B 76, 214117 (2007).

[38] P. Nordine, J. K. R. Weber, and J. G. Abadie, Pure Appl. Chem. 72, 2127 (2000).

[39] H. Fischer, G. Cuello, P. Palleau, D. Feltin, A. Barnes, Y. Badyal, and J. Simonson, Appl. Phys. A 74, s160 (2002).

[40] A. C. Barnes, S. B. Lague, P. S. Salmon, and H. E. Fischer, J. Phys. Condens. Matter 9, 6159 (1997).

[41] A. P. Hammersley, S. O. Svensson, M. Hanfland, A. N. Fitch, and D. Hausermann, High Press. Res. 14, 235 (1996).

[42] L. B. Skinner, C. J. Benmore, and J. B. Parise, Nucl. Instrum. Methods Phys. Res., Sect. A 662, 61 (2012).

[43] W. Klysubun, P. Sombunchoo, N. Wongprachanukul, P. Tarawarakarn, S. Klinkhieo, J. Chaiprapa, and P. Songsiriritthigul, Nucl. Instrum. Methods Phys. Res., Sect. A 582, 87 (2007).

[44] A. Thompson, D. Attwood, E. Gullikson, M. Howells, K. Kim, J. Kirz, J. Kortright, I. Lindau, Y. Liu, P. Pianetta, A. Robinson, 
J. Scofield, J. Underwood, and G. Williams, X-ray Bata Booklet, Centre for X-Ray Optics and Advanced Light Source (Lawrence Berkeley National Laboratory, University of California, Berkeley, CA 94720, 2001).

[45] M. Newville, J. Synchrotron Radiat. 8, 322 (2001).

[46] W. Smith and T. Forester, J. Mol. Graphics 14, 136 (1996).

[47] S. Le Roux and V. Petkov, J. Appl. Crystallogr. 43, 181 (2010).

[48] F. Farges, G. E. Brown, and J. J. Rehr, Phys. Rev. B 56, 1809 (1997).
[49] T. Tamura, S. Tanaka, and M. Kohyama, Phys. Rev. B 85, 205210 (2012).

[50] P. S. Salmon, A. C. Barnes, R. A. Martin, and G. J. Cuello, J. Phys. Condens. Matter 19, 415110 (2007).

[51] P. S. Salmon, Proc. R. Soc. London, Ser. A 445, 351 (1994).

[52] P. S. Salmon, Proc. R. Soc. London, Ser. A 437, 591 (1992).

[53] C. Angell, J. Non-Cryst. Solids 102, 205 (1988). 